\title{
A data driven deep neural network model for predicting boiling heat transfer in helical coils under high gravity
}

\author{
Xing Liang,3, Yongqi Xie ${ }^{2, *}$, Rodney Day ${ }^{3}$, Xianhai Meng ${ }^{4}$, Hongwei Wu ${ }^{3, * *}$ \\ ${ }^{1}$ School of Computing and Mathematical Sciences, University of Greenwich, SE10 9LS, UK \\ ${ }^{2}$ School of Aeronautic Science and Engineering, Beihang University, Beijing, 100191, China \\ ${ }^{3}$ School of Physics, Engineering and Computer Science, University of Hertfordshire, AL10 9AB, UK \\ ${ }^{4}$ School of Computer Science and Engineering, Beihang University, Beijing, 100191, China \\ *Corresponding Author (Prof. Yongqi Xie): Email: xyq@buaa.edu.cn Tel: +86(10)82338081

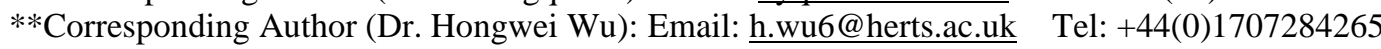

\begin{abstract}
In this article, a deep artificial neural network (ANN) model has been proposed to predict the boiling heat transfer in helical coils under high gravity conditions and compare with actual experimental data. A test rig is set up to provide the high gravity up to $11 \mathrm{~g}$ with the heat flux can be up to $15100 \mathrm{~W} / \mathrm{m}^{2}$ and the mass velocity range from 40 to $2000 \mathrm{~kg} \mathrm{~m}^{-2} \mathrm{~s}^{-1}$. In the current work, total 531 data samples have been used in the present ANN model. The proposed model was developed in Python Keras environment with Feed-forward Back-propagation (FFBP) Multi-layer Perceptron (MLP) using eight features (mass flow rate, thermal power, inlet temperature, inlet pressure, direction, acceleration, tube inner surface area, helical coil diameter) as the inputs and two features (wall temperature, heat transfer coefficient) as the outputs. The deep ANN model composed of three hidden layers with a total number of 1098 neurons and 300,266 trainable parameters has been found as optimal according to statistical error analysis. Performance evaluation is conducted based on six verification statistic metrics $\left(\mathrm{R}^{2}\right.$, MSE, MAE, MAPE, RMSE and cosine proximity) between the experimental data and predicted values. The obtained results demonstrate that 8-512-512-64-2 neural network model has the best performance in predicting the helical coil characteristics with $\left(\mathrm{R}^{2}=0.853, \mathrm{MSE}=0.018, \mathrm{MAE}=0.074, \mathrm{MAPE}=1.110\right.$, $\mathrm{RMSE}=0.136$, cosine proximity $=1.000$ ) in testing stage. It is indicated that with the utilisation of deep learning, the proposed model is able to successfully predict the heat transfer performance in helical coils, especially achieved excellent performance in predicting the outputs having very large range of value differences.
\end{abstract}

KEY WORDS: Boiling, heat transfer, helical coils, deep neural network, high gravity

\section{INTRODUCTION}

Helical coils have attracted wide attention due to their high heat transfer efficiency, compact design, ease of manufacture and freedom from thermal deformation [1,2]. Over the past two decades, helical coils have been widely used in energy and cooling systems such as air conditioning [3,4], heat exchanger [5-7], steam generators [8,9], renewable energy[10,11] and energy storage [12,13], chemical and petroleum industries $[14,15]$. In helically coiled tubes, the centrifugal force induced by the oil geometry will exert upon the working fluid, which will lead to the second flows (Dean vortices) $[16,17]$. It is recognized that flow boiling heat transfer represents one of the most efficient type of heat transfer mode. The mechanism of flow boiling heat transfer inside a helical coil may be different compared with that in a straight tube due to secondary flows generated inside a helical coil [18].

In recent years, due to its practical relevance, many research efforts have been devoted to the flow and boiling heat transfer in helically coiled tubes. This paper does not and cannot review all the interesting and important progress related to boiling heat transfer in helical coils, but tries to summarize the recently published results in the concerned field. Santini et al. [19] experimentally 
investigated the forced convection boiling of water in an electrically heated, $24 \mathrm{~m}$ long full-scale helically coiled steam generator tube with the operating pressures in the range of 2-6 MPa, mass fluxes from 200 to $800 \mathrm{~kg} \mathrm{~m}^{-2} \mathrm{~s}^{-1}$ and heat fluxes from 40 to $230 \mathrm{~kW} \mathrm{~m}$. Their results showed that the heat transfer coefficient was found to be depend on the mass flux and heat flux, indicating that both nucleate boiling and convection were contributing to the heat transfer. It also noted that the effect of curvature on the heat transfer during flow boiling could be negligible and therefore the heat transfer coefficient in helical coils could be predicted with existing correlations for straight tubes. Later on, Tan et al. [20] conducted an experimental study to investigate critical heat flux characteristics in a uniformly heated horizontal helically coiled tube at near the critical pressure ranges from 2.85 to 4.03 $\mathrm{MPa}$ with R134a as the working fluid. There were two types of critical heat flux phenomenon which were dry-out and departure from nucleate boiling were found from their experimental study. The dryout occurred at the lower pressure while the departure from nucleate boiling happened at the higher pressure. Kong et al. [21] performed an experiment work to investigate the subcooled boiling heat transfer characteristics of R134a in vertical helically coiled tubes with the operating pressures varying from 450 to $850 \mathrm{kPa}$, mass fluxes from 147.5 to $443.7 \mathrm{~kg} \mathrm{~m}^{-2} \mathrm{~s}^{-1}$ and heat fluxes from 0.1 to $14.5 \mathrm{~kW}$ $\mathrm{m}^{-2}$. Their experimental results showed that the generated bubbles always slide along the heated surface in the vertical helically coiled tube. It was also found that the radial component force is negative, which contributes significantly to the heat transfer enhancement and nonuniformity of the wall temperature in subcooled flow boiling. Hardik and Prabhu [22] performed an experimental investigation to study the effect of curvature on local boiling heat transfer coefficient and two-phase pressure drop in helically coiled tubes with water as the working fluid. They concluded that in the subcooled region and in a low quality region, boiling heat transfer coefficient in helical coils will be much higher (12\%-100\%) than in straight tube, whereas in a high quality region, the boiling heat transfer coefficient in will be the same as in straight tubes. In addition, they developed a correlation for two-phase pressure drop in helical coils. Afterwards, the same authors [23] experimentally investigated the characteristics of the boiling heat transfer, pressure drop and critical heat flux for refrigerant R123 with six helical coils having three different tub diameters and two different coil diameters. They concluded that in fully developed flow boiling region, the circumferential averaged heat transfer coefficient in helical coils is same as in straight tubes. Xiao et al. [24] carried out various experiments in helically coiled tubes to explore the boiling heat transfer characteristics at high pressure with water as the working medium with the pressure ranges from 2 to $8 \mathrm{Mpa}$. Their experimental results indicated that the subcooled boiling and saturated nucleate boiling heat transfer coefficient would increase with the increase of heat flux, whereas heat flux showed no influence on saturated convective boiling heat transfer coefficient. The effects of mass flux and system pressure on heat transfer coefficients were also discussed. Niu et al. [25] developed an analytic model based on liquid film dryout theory to predict the onset of dryout quality in annular flow regime in helically coiled tubes and validated against previously published experimental data. It was found that the onset of dryout quality decreased as mass flux increased in the re-deposition-dominated region, and the total net entrainment rate increased gradually with the increase of heat flux. Chen et al. [16] experimentally studied the heat transfer characteristics of subcooled flow boiling in helical coils with different inner diameter and coil diameters. The concluded that the heat transfer coefficients in subcooled boiling regions can be improved by increasing heat flux, mass flux and system pressure. Sun et al. [26] proposed a two-fluid model taking the vapour phase and the liquid phase into account in order to capture the non-uniform wall temperature distribution of boiling heat transfer in a helically coiled tube. Their simulation results showed that the liquid accumulated at the outside and bottom of the cross section during nucleate boiling heat transfer, leading to a lower wall temperature. Hernández-Magallanes and Rivera [27] reported the experimental data of boiling heat transfer coefficients for the ammonia-lithium nitrate mixture in a laminar falling film. They found that the ammonia vapour could be produced at generation temperatures between $80{ }^{\circ} \mathrm{C}$ and $105^{\circ} \mathrm{C}$ obtaining boiling heat transfer coefficients between 85 and $340 \mathrm{~W} / \mathrm{m}^{2} \mathrm{~K}$. Most recently, Shaver et al. [28] 
performed a numerical study of the boiling flow inside a helical coil with a two-fluid model using the spectral element code Nek-2P. Their simulation results indicated that a strong phasic separation driven by the effects of buoyancy and inertia.

From the literature review above, it can be concluded that most of the work on the boiling heat transfer in helical coil mainly focused on experimental investigation and only quite few studies on numerical simulation. In addition, all the above research work were investigated under terrestrial gravity. However, it should be recognized that helical coils can be used as one of the heat transfer enhancement techniques in aerospace which will experience a high gravity stage. High gravity conditions would make the boiling flow and heat transfer more complicated and make the experiment more difficult $[29,30]$. Thus, to investigate the effects of the elevated high gravity on the boiling flow and heat transfer is very important and challenging. Most recently, Bai et al. [31] carried out a numerical study on heat transfer of supercritical $\mathrm{CO}_{2}$ in heated helically coiled tubes under abnormalgravity such as high-gravity, low-gravity and zero-gravity in order to explore the coupled mechanisms of buoyancy and centrifugal force. Their study did not consider the phase change. It was found that the heat transfer coefficient could be enhanced at higher gravity force in the liquid-like region, while it could be almost the same in the gas-like region. They also stated that the heat transfer could be enhanced by buoyancy force when the gravity is higher than terrestrial gravity, whereas the heat transfer could be weakened under lower gravity. It needs to be stressed here that, under high gravity, extra forces such as centrifugal force and Coriolis force will make the phenomena more complicated as well as for boiling flow and heat transfer the two-phase flow will make the computational fluid dynamic (CFD) simulation more difficult.

Deep learning neural networks with multiple layers between the input and output layer can model complex non-linear relationships. Most recently, a deep neural network named physics-informed neural networks is able to model nonlinear partial differential equation characteristics and synergy between machine learning and classical computational physics [32-34]. Machine learning (ML) artificial neural networks (ANNs) are not new in concept, still they have received considerable attention and they have been applied successfully in a wide range of fields for modelling and prediction in boiling flow and heat transfer [35-40] but very little work on helical coils. Gill and Singh [41] developed both dimensionless correlation and ANN models to predict the mass flow rate of R134a/LPG refrigerant through straight and helical coil adiabatic capillary tubes in a vapor compression refrigeration system. It was found that the ANN model gave better statistical prediction than dimensionless correlation model. Parrales et al. [42] developed two new empirical equations based on ANN to determine the new void fraction in two-phase flow inside helical vertical coils with water as work fluid. The analyses of the contribution of input variables in the ANN model showed that the curvature ratio could not impact the simulative accuracy of void fraction under the current conditions. Izadi et al. [43] employed ANN and adaptive neuro-fuzzy inference system (ANFIS) models to evaluate micromixing in micro-helically coiled tubes. It was found that ANN approach has higher precision than ANFIS in segregation index modelling in micro-helically coiled tubes. Baghban et al. [44] proposed a machine learning approach with three different models such as multilayer perceptron artificial neural network (MLP-ANN), adaptive neuro-fuzzy inference system (ANFIS), and least squares support vector machine (LSSVM) to predict Nusselt number by considering Prandtl number, volumetric concentration, and helical number of helically coiled heat exchanger as input variables, as well as using water carbon nanofluid as the working fluid. Their results showed that LSSVM approach has the best performance. Most recently, Sharifi et al. [45] performed a three dimensional numerical simulation to investigate the effect of coiled wire inserts with various arrangements on friction coefficient and heat transfer rates for the helical wire inserted tube in heat exchangers. They stated that, with the assist ANN, the heat transfer rates and the friction coefficients could be accurately estimated within the ranges of input variables. 
In the view of the aforementioned literature survey, many significant results in the boiling flow and heat transfer characteristics of helical coils have already been obtained. However, lack of tests dealing with the flow and heat transfer in helical coils under high gravity. To the best of the authors' knowledge, ANN methods have not yet been applied to that thermal system. In the current work, a data set is obtained experimentally and prepared for processing with the use of ANNs. A feed-forward back-propagation multi-layer perceptron deep ANN is proposed to predict the heat transfer coefficient in order to better understand the boiling flow and heat transfer mechanism as well as for future design and analysis.

\section{EXPERIMENTAL APPARATUS AND PROCEDURE}

\subsection{Experimental apparatus}

In the current work, the actual details of the helical coils heat transfer test rig was consistent with the previous study of Xie et al. [29] and designed to be operated under high gravity conditions up to 11g, and will only be described briefly here for the sake of completeness. The test rig mainly consists of a centrifuge in a circular pit, liquid circulation system, test section, control and data acquisition system. Fig. 1 showed the schematic diagram of the experimental apparatus. A thermostatic water tank was used to keep the working fluid (R601 n-pentane) temperature constant. Fluid flows was driven by a gear pump through a variable-frequency drive under high gravity and a Coriolis force flow meter was chosen to measure the mass flow rate with an accuracy of $\pm 5 \%$. After the working fluid flowed through the mass flow meter, a preheater was used to heat up the fluid to the required temperature at the test section inlet. The test section coils that connected across two cooper electrodes were used as the electrical resistance. They were connected to a DC power supply in order to deliver the heating power to the flowing fluid. The working fluid came out from the helical coils will be entered a condenser where it was cooled to a low temperature before recycling back to the thermostatic tank. All the signals measured by the temperature and pressure transducers were achieved by utilizing digital interface and recorded by a remote computer. In the current study, it was noted that the centrifuge driven by an electric motor can provide up to $15 \mathrm{~g}$ rotational acceleration at the end of the rotating arm. The rotational speed of the centrifuge could be well controlled by a control terminal with an accuracy of $\pm 5 \%$ the indicated speed.

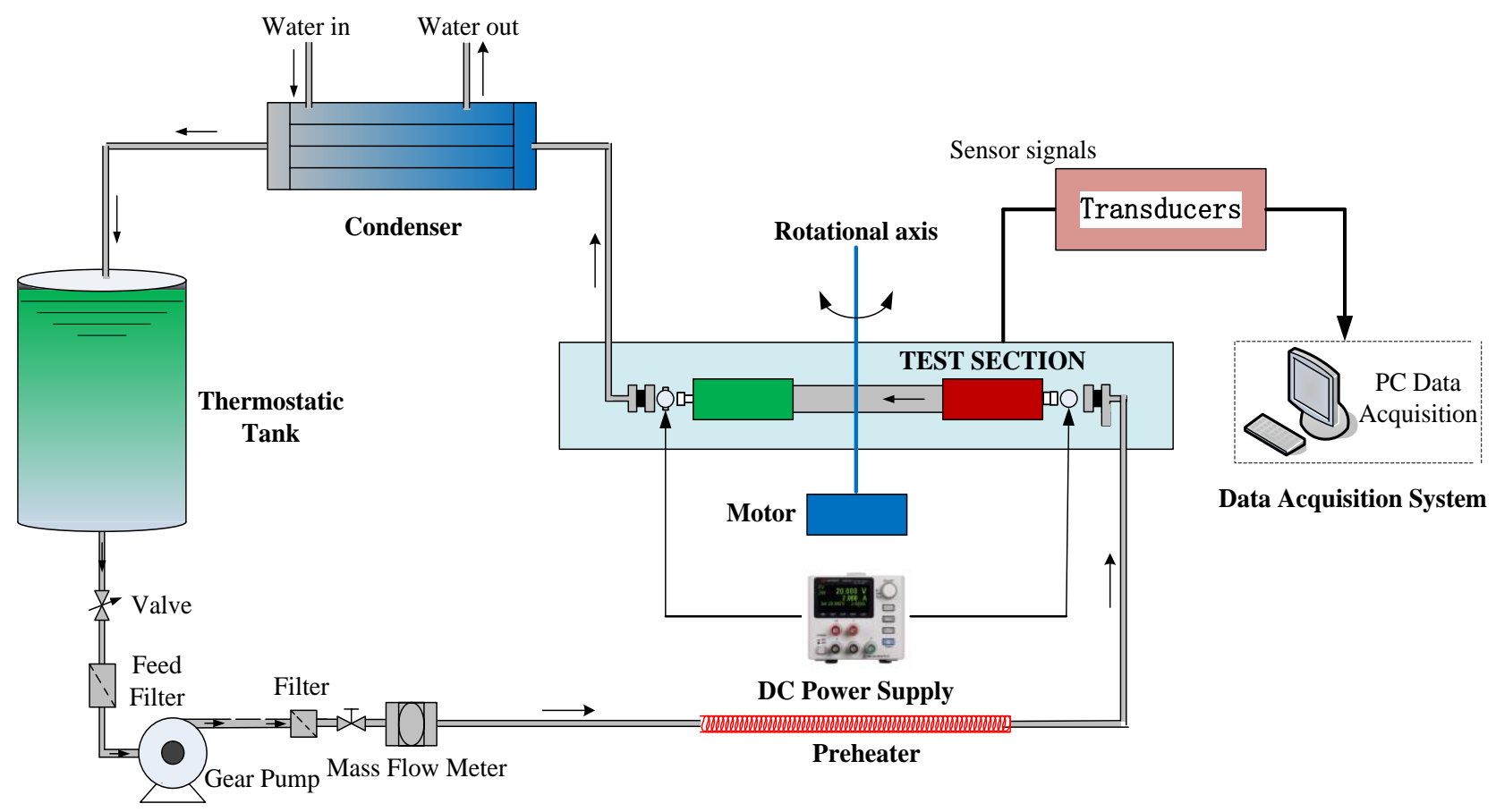

Fig. 1. Schematic diagram of experimental apparatus. 


\subsection{Test section and procedure}

During the rotating condition, the test section was fixed in order to obtain the stead flow boiling condition. The stainless steel helical coils were electrified by a power supply to heat the working fluid. In the present study, R601 (n-pentane) was selected as the working fluid with a low saturation temperature of approximately $36^{\circ} \mathrm{C}$ at atmosphere pressure. A layer of the glass wool acted as an insulator to minimize the heat losses of the coils. Fig. 2 showed the schematic diagram of the helical coils. The helical coil material and geometrical parameters were carefully designed so that its electric resistance could fit the power supply and the maximum heating power could be reached. The fluid temperatures at both the inlet and outlet of the coils were measured by a direct immersion Pt100 thermo-resistor. Fig. 2 also showed that the wall temperature position of the Pt100 thermo-resistor attached to the outer surface of the coil. Both the inlet and outlet fluid pressures were measured using two Trafag pressure transducers. For the purpose of accuracy, all the sensors were calibrated prior to each test. Table 1 listed the geometrical parameters of the helical coils and Table 2 listed the specifications of all the instruments. Two directional (axial and radial) configurations (see Fig. 3) and three different acceleration (3, 6 and $9 \mathrm{~g}$ ) were applied. The experimental conditions were summarized in Table 3.

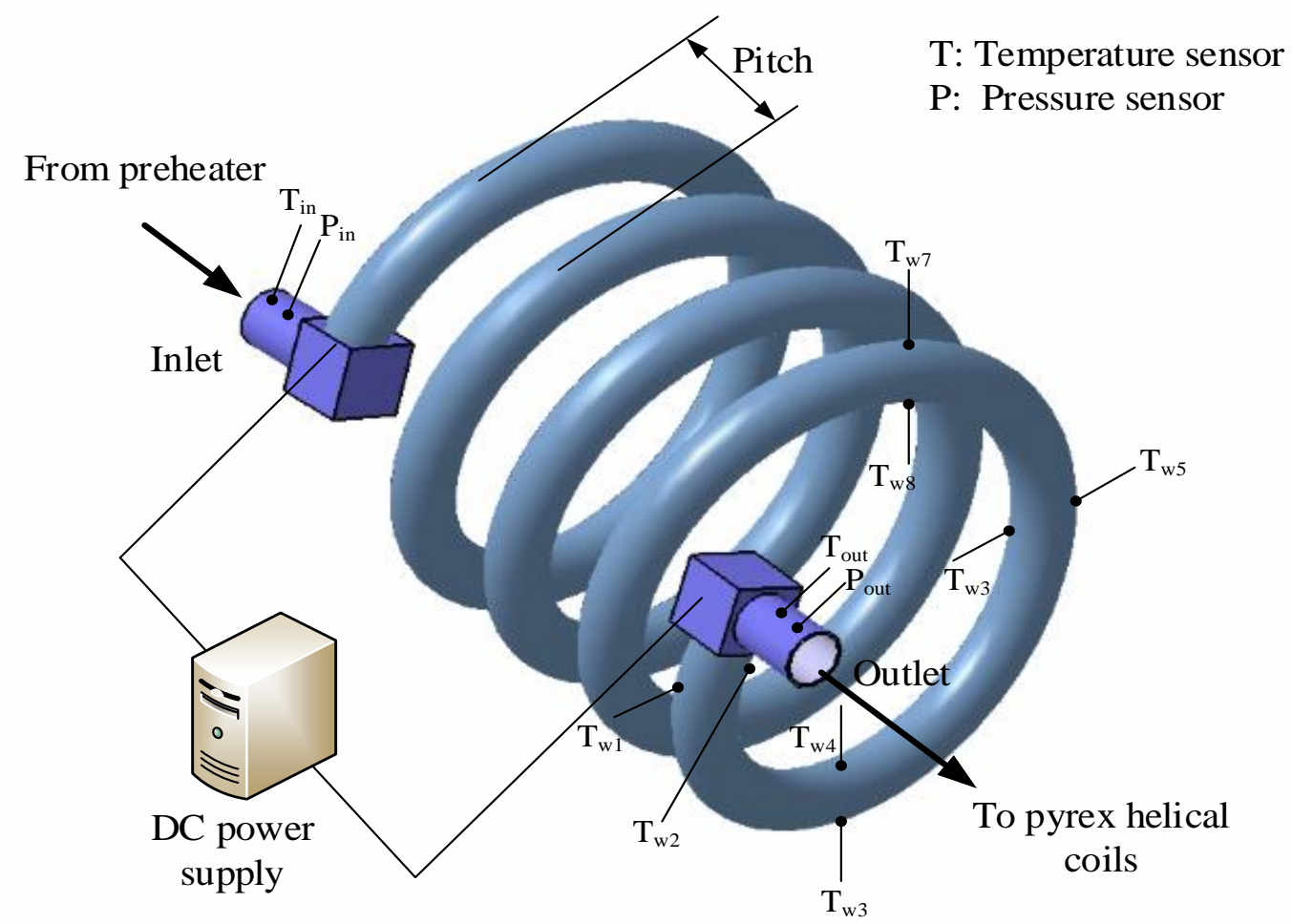

Fig. 2. Test section (helical coils and transducer allocation).

Table 1 Geometrical parameters of the helical coils.

\begin{tabular}{c|c|c|c|c|c|c|c}
\hline $\begin{array}{c}\text { Coil } \\
\text { No. }\end{array}$ & $\begin{array}{c}\text { Tube inner } \\
\text { diameter, } \\
d_{i}(\mathrm{~mm})\end{array}$ & $\begin{array}{c}\text { Tube outer } \\
\text { diameter, } \\
d_{o}(\mathrm{~mm})\end{array}$ & $\begin{array}{c}\text { Coil } \\
\text { diameter, } \\
D_{\text {coil }}(\mathrm{mm})\end{array}$ & $\begin{array}{c}\text { Pitch, } \\
P(\mathrm{~mm})\end{array}$ & $\begin{array}{c}\text { Tube } \\
\text { length, } \\
L(\mathrm{~m})\end{array}$ & $\begin{array}{c}\text { Tube inner } \\
\text { surface area, } \\
S\left(\mathrm{~mm}^{2}\right)\end{array}$ & $\begin{array}{c}\text { Circling } \\
\text { laps, } \\
N\end{array}$ \\
\hline 1 & 8.9 & 9.5 & 115 & 30 & 2.46 & 68741 & 7.0 \\
2 & 8.9 & 9.5 & 135 & 30 & 2.33 & 65238 & 5.5 \\
3 & 8.9 & 9.5 & 150 & 20 & 2.35 & 65844 & 5.0 \\
4 & 8.9 & 9.5 & 150 & 30 & 2.36 & 65906 & 5.0 \\
5 & 8.9 & 9.5 & 150 & 35 & 2.36 & 65920 & 5.0 \\
6 & 8.9 & 9.5 & 170 & 30 & 2.40 & 67184 & 4.5 \\
\hline
\end{tabular}




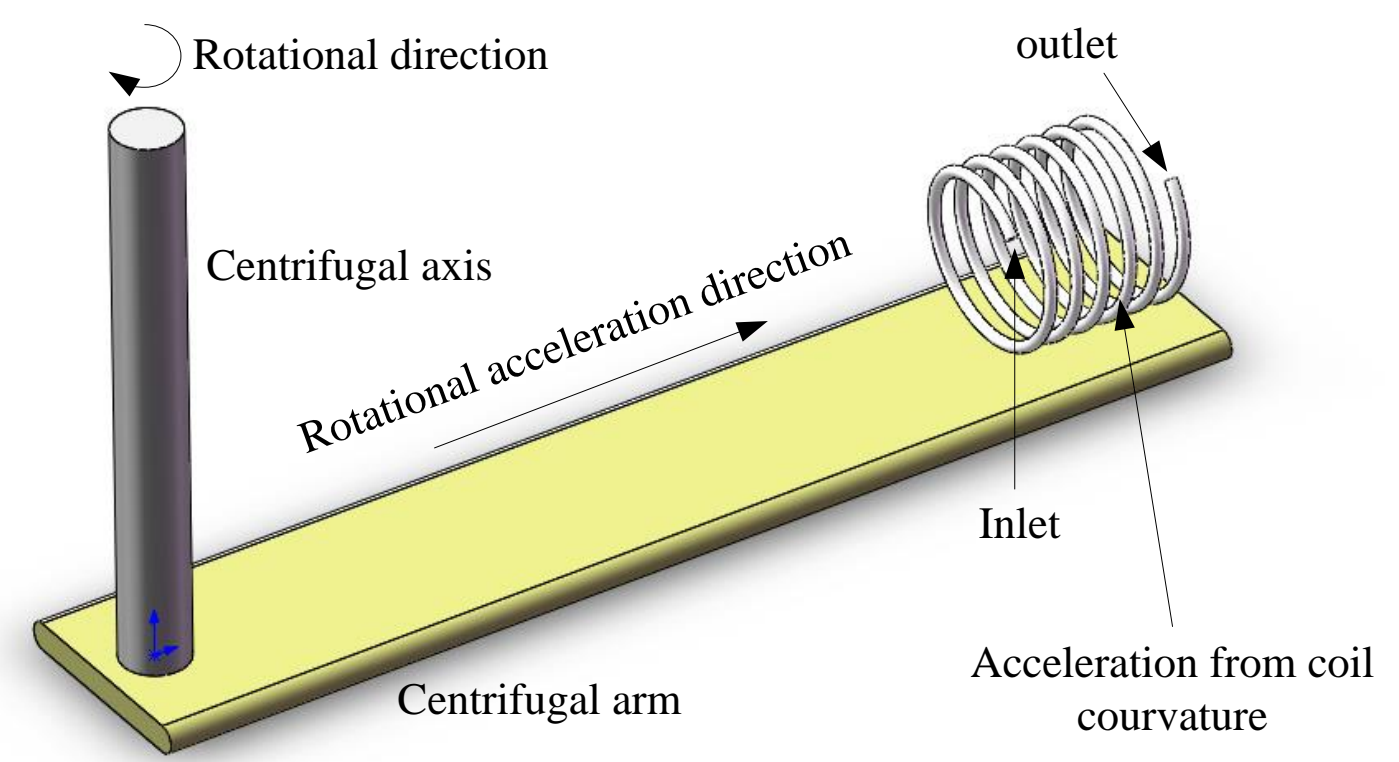

(a)

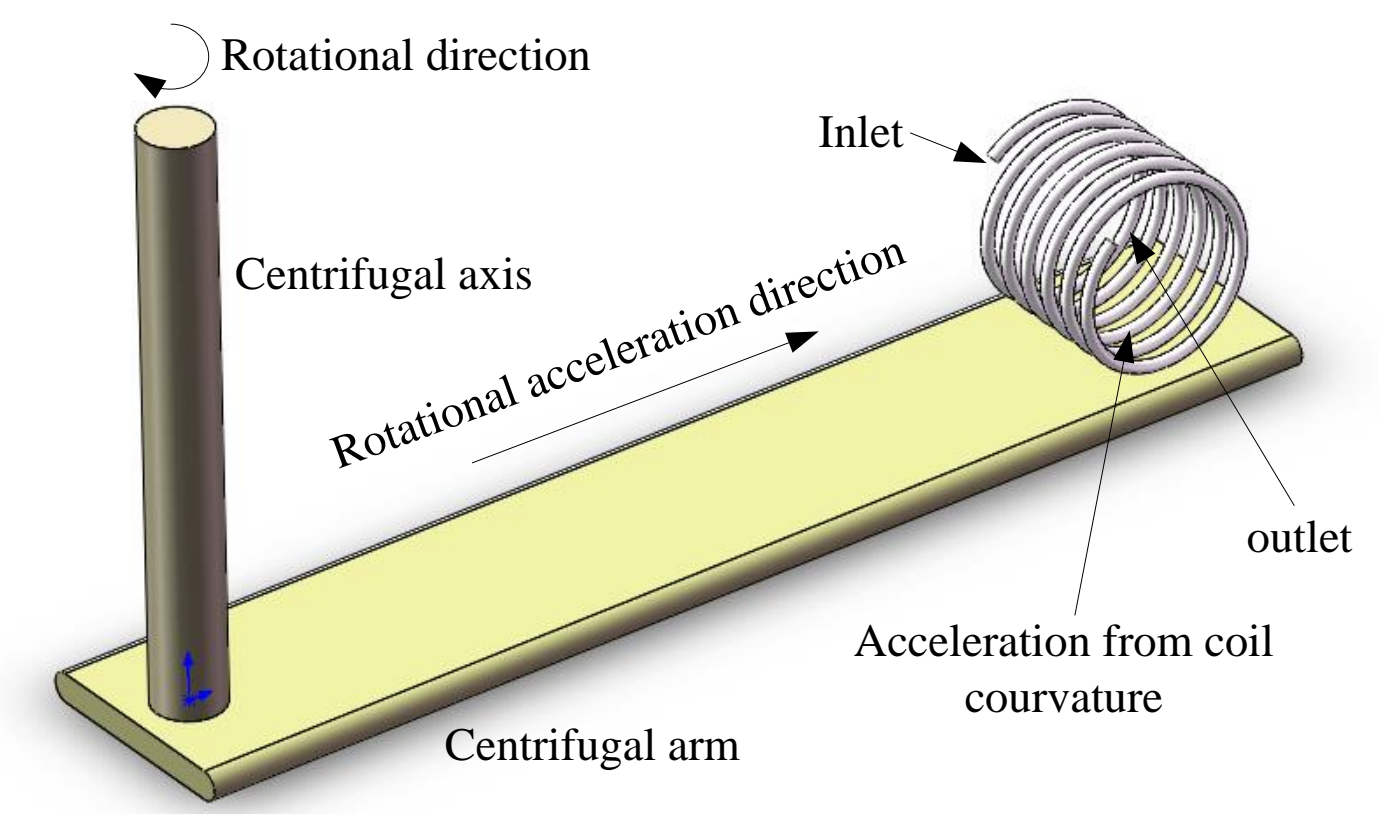

(b)

Fig. 3. Configurations for acceleration direction (a) axial and (b) radial.

Table 2 Instrument specifications.

\begin{tabular}{c|c|c}
\hline Item & Range & Maximum accuracy \\
\hline Pt100 thermo-resistor & $-50{ }^{\circ} \mathrm{C}$ to $150{ }^{\circ} \mathrm{C}$ & $0.5 \mathrm{~K}$ \\
Mass flow meter & $5-50 \mathrm{~kg} / \mathrm{h}$ & $0.25 \mathrm{~kg} / \mathrm{h}(0.5 \%$ full scale $)$ \\
Pressure transducer & $0-160 \mathrm{kPa}$ & $0.8 \mathrm{kPa}(0.5 \%$ full scale $)$ \\
Power supply & $30 \mathrm{~V}, 100 \mathrm{~A}$ & $0.3 \mathrm{~V}, 0.5 \mathrm{~A}$ \\
\hline
\end{tabular}


Table 3 Experiment conditions.

\begin{tabular}{c|c|c}
\hline Parameter & Symbol/unit & Range \\
\hline Heat flux & $q / \mathrm{W} \mathrm{m} \mathrm{m}^{-2}$ & $0-15100$ \\
Inlet pressure & $P_{\text {in }} / \mathrm{kPa}^{-2}$ & $120-200$ \\
Mass velocity & $G / \mathrm{kg} \mathrm{m}^{-1} \mathrm{~s}^{-1}$ & $40-200$ \\
Outlet quality & $x_{\text {out }} / \%$ & $0-50$ \\
Start-up time & $t_{s} / \mathrm{min}$ & 1 \\
Acceleration duration time & $t_{d} / \mathrm{min}$ & 3 \\
\hline
\end{tabular}

\section{HEAT LOSS, DATA REDUCTION AND UNCERTAINTY ANALYSIS}

\subsection{Heat loss calculation}

In the current work, the test section was mounted on the rotational arm and the DC power supply was located in the control terminal outside the pit, there was voltage drop between the control terminal and the test section. This voltage drop loss can be accurately calculated through measuring the voltage between the coils since the electric resistance of the electric wires cannot be neglected due to the wire length. In addition, the conduction from the heating wall to the environment contributed to the heat losses. In a conduction-convection system the heat losses are proportional to the temperature difference between the test rig and the ambient. The heat loss, as a function of average wall temperature and ambient temperature, can be calculated using Eq. (1), and will be applied in the subsequent boiling experiments to calculate the heat losses.

$$
Q_{\text {loss }}=0.480\left(T_{w, a v g}-T_{a m b}\right)
$$

where $Q_{l o s s}$ is the heat losses, $T_{w, a v g}$ is the average wall temperature and $T_{a m b}$ is the ambient temperature.

\subsection{Data reduction and uncertainty analysis}

The heat flux from the coil wall delivered to the fluid was calculated as follows:

$$
q=Q_{\text {eff }} / A=\left(V I-Q_{\text {loss }}\right) / A
$$

where $q$ is the heat flux delivered to the fluid, $Q_{\text {eff }}$ is the effective heating power delivered to the fluid, $A$ is coil inner surface area, $V$ and $I$ are the DC voltage and current on the coils.

The boiling heat transfer coefficient under high gravity can be calculated as:

$$
h_{\text {out }}=q /\left[0.5\left(T_{w 1, g}+T_{w 2, g}\right)-T_{s a t, g}\right]
$$

where $T_{s a t}$ is the saturated temperature calculated from the outlet pressure, $T_{w}$ is the coil wall temperature at inlet and outlet. The subscript $g$ indicates the high gravity.

The thermodynamic vapor quality can be calculated as follows:

$$
x_{g}=\left[q A-c_{p} \dot{m}\left(T_{s a t, g}-T_{i n, g}\right)-T_{s a t}\right] / \dot{m} i
$$

where $c_{p}$ is the specific heat of the local bulk of the fluid, $\dot{m}$ is the mass flow rate and $i$ is the vaporization latent heat.

The pressure drop can be calculated as follows: 


$$
\Delta p_{g}=p_{\text {in }, g}-p_{\text {out }, g}
$$

where $p_{\text {in }}$ and $p_{\text {out }}$ are the inlet and outlet pressure of the helical coils.

All the properties of the pentane are acquired from NIST REFPROP directly without any secondary interpolation [46]. The geometric parameters of the coils were machined with an accuracy of \pm 0.5 $\mathrm{mm}$. The accuracies of the thermocouples, pressure sensors, flowmeter and power supply can be found in Table 2. Following the error transfer function [47], the typical uncertainties of the key parameters were summarized in Table 4.

Table 4 Parameter uncertainties.

\begin{tabular}{c|c}
\hline Parameter & Typical uncertainty (\%) \\
\hline Heat flux & 11.2 \\
Heat transfer coefficient & 13.2 \\
Vapor quality & 15.7 \\
Pressure drop & 2.8 \\
\hline
\end{tabular}

\section{Artificial neural network modelling}

In recent years, machine learning has been widely applied to solve various of problems such as classification, regression, clustering, reinforcement learning, computer vision and natural language processing. Deep neural networks with three or more hidden layers is observed to have higher generalisation capability compared to shallow neural networks. We observed the use of deep neural network as a nonlinear regression model to estimate the complex characteristic of boiling heat transfer in helical coils under high gravity. Considering that the output prediction on boiling heat transfer coefficient and outlet temperature falls into large scale range differences under current operating condition, supervised deep learning ANN with more neurons and hidden layers are necessary for learning the compound behaviours of boiling heat transfer in helical coils from experimental datasets.

\subsection{Dataset and data pre-processing}

This section discusses on the technical details of the data pre-processing in our ANN approach to increase the model performance accuracy. We conduct a detailed statistical analysis and exploration to understand the dataset, remove duplicates, treat errors and outliers, scale the features and split the dataset. To estimate the boiling heat transfer in helical coils under high gravity, 531 labelled datasets collected from experiments in .csv format are used for model training process. Each record in the dataset is represented as a vector of 10 attributes, including mass flow rate, thermal power, inlet temperature, inlet pressure, direction, acceleration, tube inner surface area, helical coil diameter, wall temperature, and heat transfer coefficient.

Correlation matrix heatmap is generated with the purpose of attribute visualization and feature engineering, as feature selection is an important step in building a regression model. Correlation analysis evaluates the strength of relationship between two quantitative attributes. A high correlation means that the attributes have a strong relationship with each other, while a low correlation means that the attributes are hardly related. As shown in Fig. 4. the correlation analysis results in eight unique input features: mass flow rate, thermal power, inlet temperature, inlet pressure, direction, acceleration, tube inner surface area, and helical coil diameter, which are more in correlation with the outputs features: wall temperature, heat transfer coefficient. The eight input features are further pre-processed before feed into the proposed ANN model. 


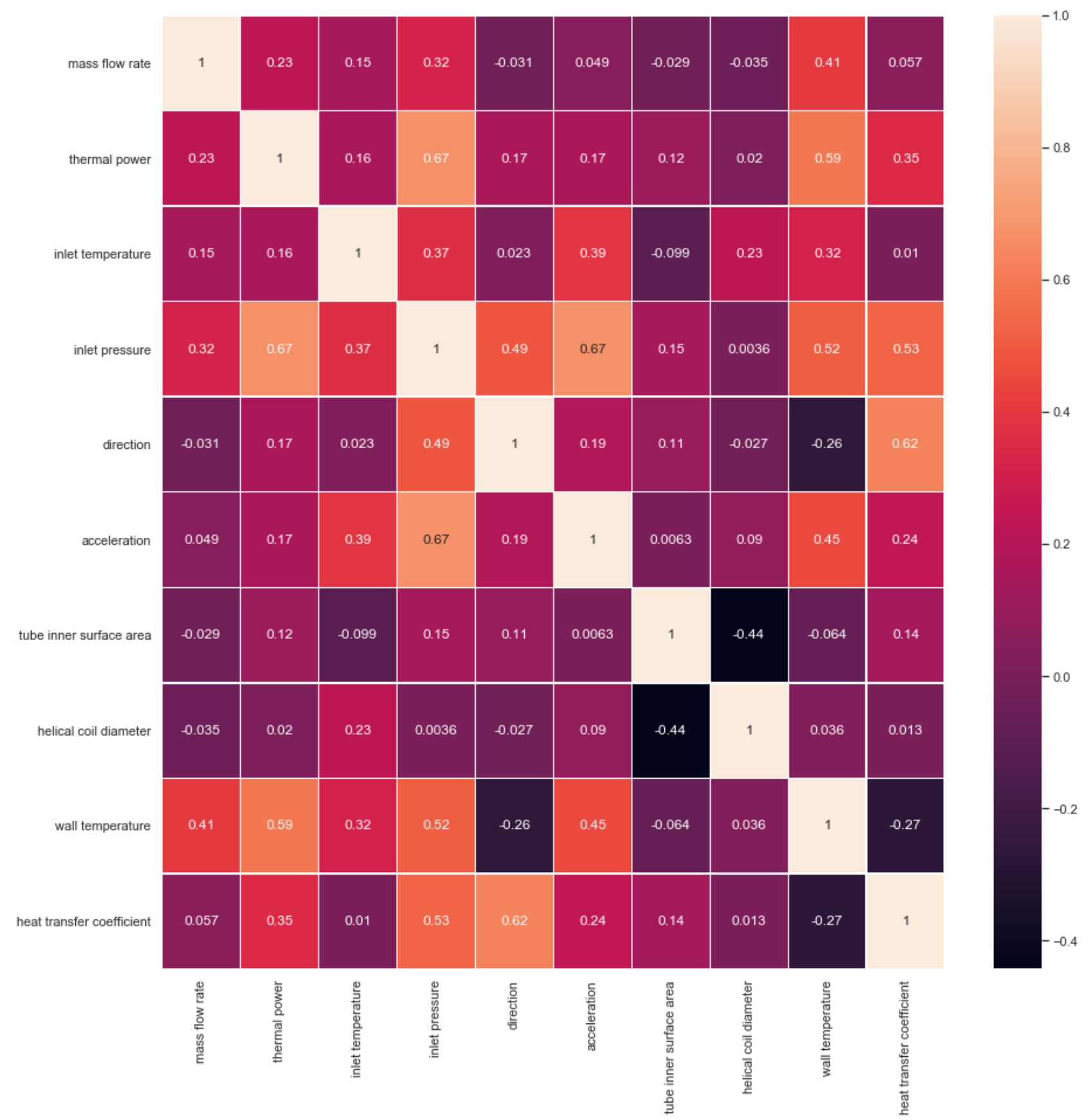

Fig. 4. Attributes correlation matrix heatmap.

With the selected features, a statistical analysis is conducted to monitor feature-wise values for minimum, maximum, and standard deviation. Outliers are detected and bad working points are removed by setting a max and min range of credible values and limit to that. All features in the dataset are in either numeric value as continuous measurement data or nominal values to represent categorical measurement condition. Mass flow rate, thermal power, inlet temperature, inlet pressure are considered as numeric input features; direction, acceleration, tube inner surface area, helical coil diameter are considered as categorical input features. Consequently, different type of normalisation or feature scaling would be necessary for numeric and categorical input features prior to training process. 
Table 5 Explanation of input and output features.

\begin{tabular}{l|l|l}
\hline Inputs and outputs & \multicolumn{1}{|c|}{ Attribute form in model } & \multicolumn{1}{c}{ Range } \\
\hline \multirow{4}{*}{ Inputs } & mass flow rate $(\mathrm{kg} / \mathrm{s})$ & $14.00-40.86$ \\
\cline { 2 - 3 } & thermal power $(\mathrm{W})$ & $173.89-995.12$ \\
\cline { 2 - 3 } & inlet temperature $\left({ }^{\circ} \mathrm{C}\right)$ & $27.95-32.46$ \\
\cline { 2 - 3 } & inlet pressure $(\mathrm{kPa})$ & $23.73-87.41$ \\
\cline { 2 - 3 } & direction & $0(\mathrm{axial}), 1(\mathrm{radial})$ \\
\cline { 2 - 3 } & Acceleration & $0(3 \mathrm{~g}), 1(6 \mathrm{~g}), 2(9 \mathrm{~g})$ \\
\cline { 2 - 3 } & tube inner surface area $\left(\mathrm{mm}^{2}\right)$ & $65350.57-68891.88$ \\
\cline { 2 - 3 } & helical coil diameter & $0(115 \mathrm{~mm}), 1(130 \mathrm{~mm}), 2(135 \mathrm{~mm})$, \\
& & $3(150 \mathrm{~mm}), 4(170 \mathrm{~mm}), 5(190 \mathrm{~mm})$ \\
\hline \multirow{2}{*}{ Outputs } & wall temperature $\left({ }^{\circ} \mathrm{C}\right)$ & $43.53-69.35$ \\
\cline { 2 - 3 } & heat transfer coefficient $\left(\mathrm{W} / \mathrm{m}^{2} \mathrm{~K}\right)$ & $701.09-5849.37$ \\
\hline
\end{tabular}

Table 5 summarises the denoted symbols, descriptions and ranges of all attributes utilised for the model. It is clearly indicates that the scales of the numeric features on the dataset are heavily imbalanced. For example, a typical inlet temperature corresponds to a float value range from $27.95{ }^{\circ} \mathrm{C}$ to $32.46{ }^{\circ} \mathrm{C}$, while a typical of thermal power to a range from $173.89 \mathrm{~W}$ up to $995.12 \mathrm{~W}$.
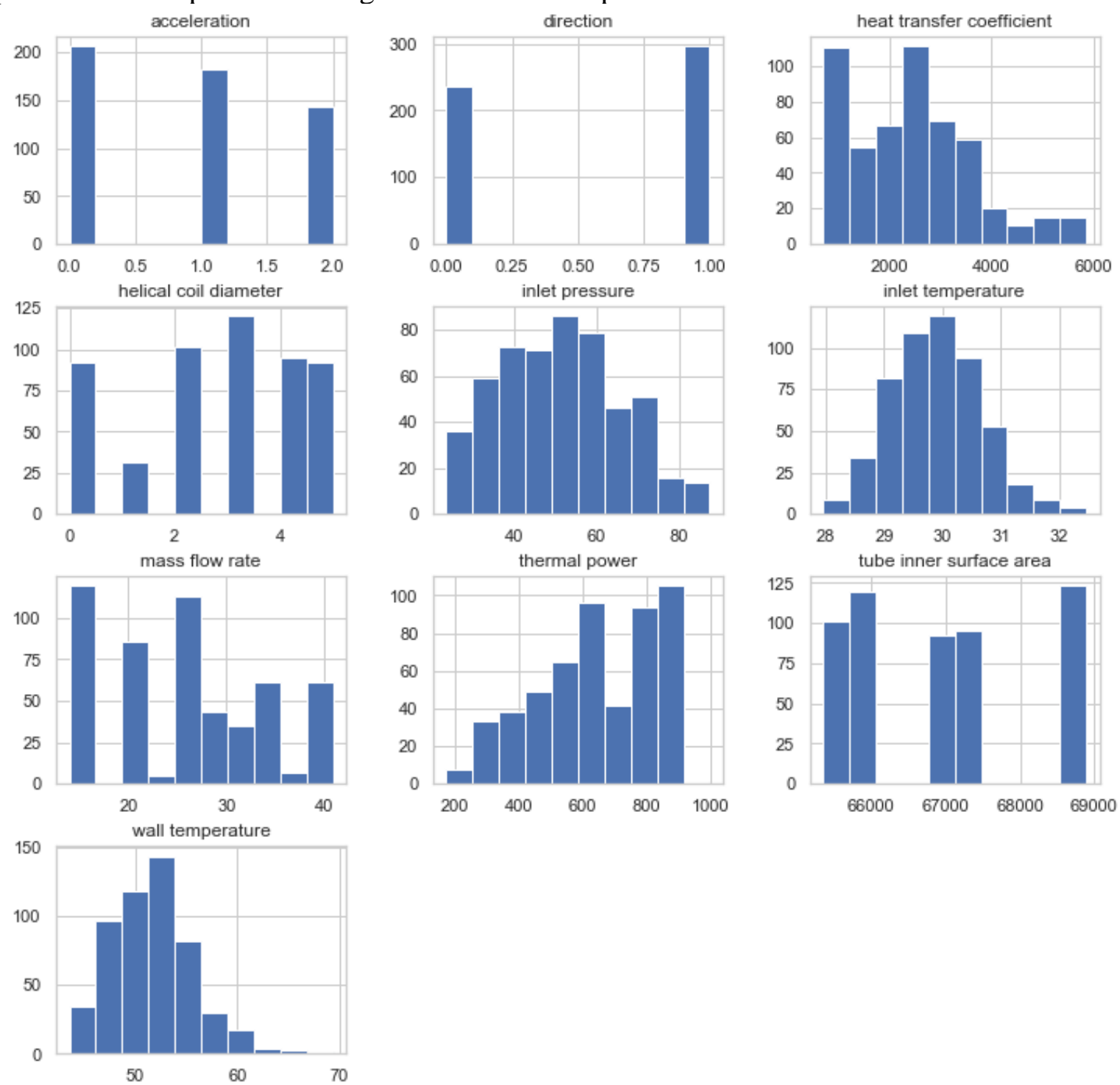

Fig. 5. Original feature visualisation with histogram. 
Additionally, some features such as thermal power in Fig. 5 with significant skewness can have an impact on prediction models. Therefore, in the current work, all numeric features are transformed by the approach of the Box-Cox. In order to clearly present how feature scaling is applied to numeric inputs, Fig. 6 depicts the original input feature and the corresponding Box-Cox feature scaling. On the other hand, as demonstrated in Fig. 7, nominal input feature vectors are converted into categorical features and Z-score scaling is applied. Furthermore, since there are very large discrepancies of values between wall temperature (value range: $43.53-69.35{ }^{\circ} \mathrm{C}$ ) and heat transfer coefficient (value range: 701.09-5849.37 $\mathrm{Wm}^{-2} \mathrm{~K}^{-1}$ ), logarithmic scaling is applied to output features and inverse transform is performed during model performance evaluation (see Fig. 8).

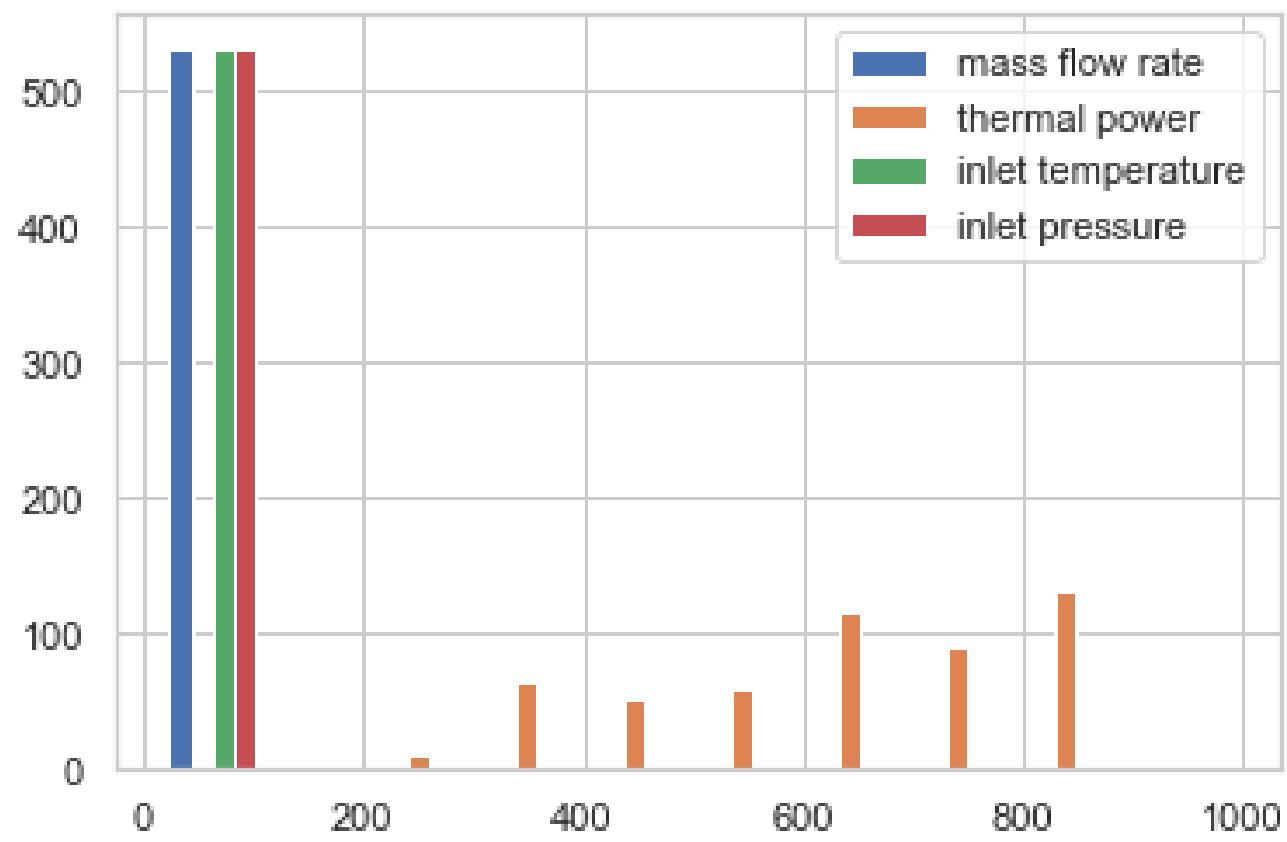

(a) Original numeric feature

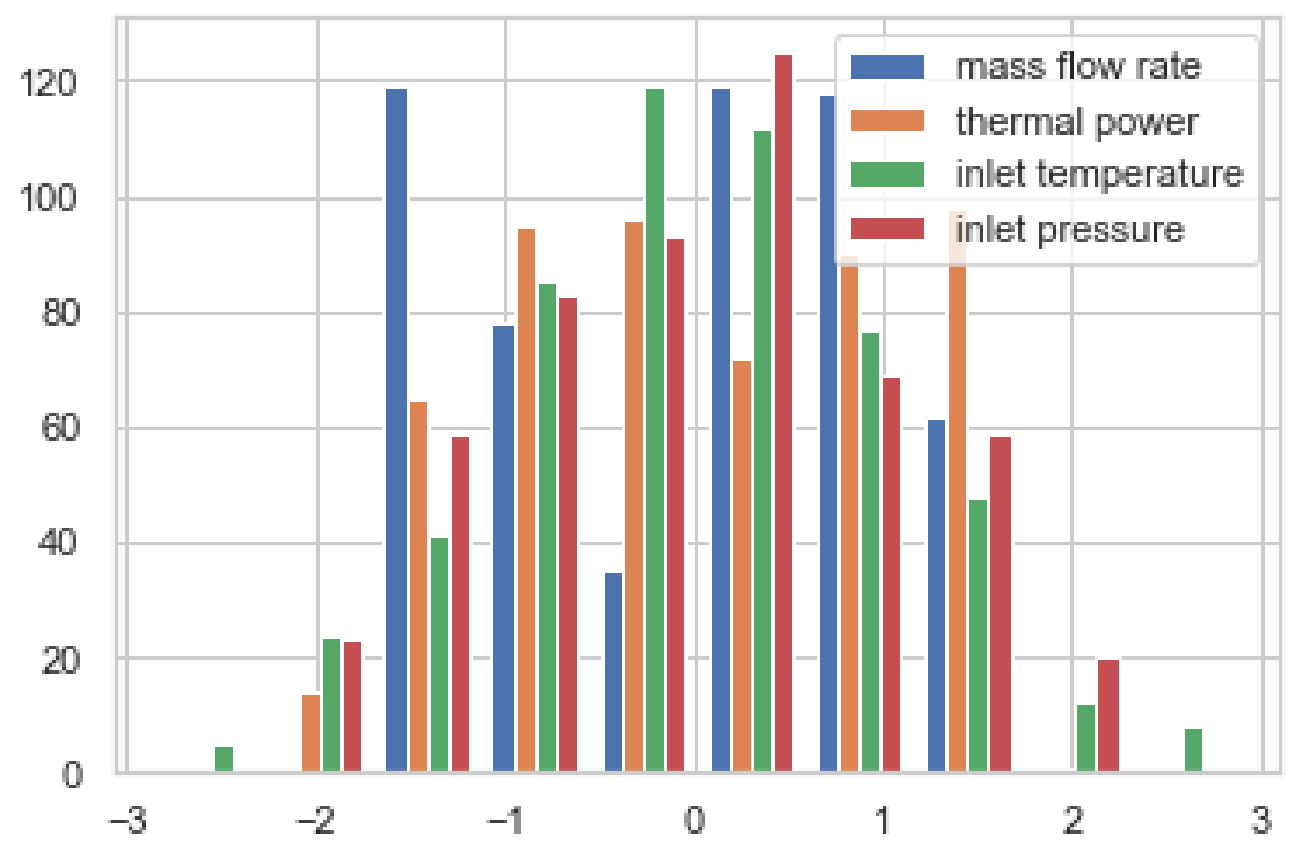

(b) Numeric feature after box-cox scaling

Fig. 6. Numeric input feature scaling. 

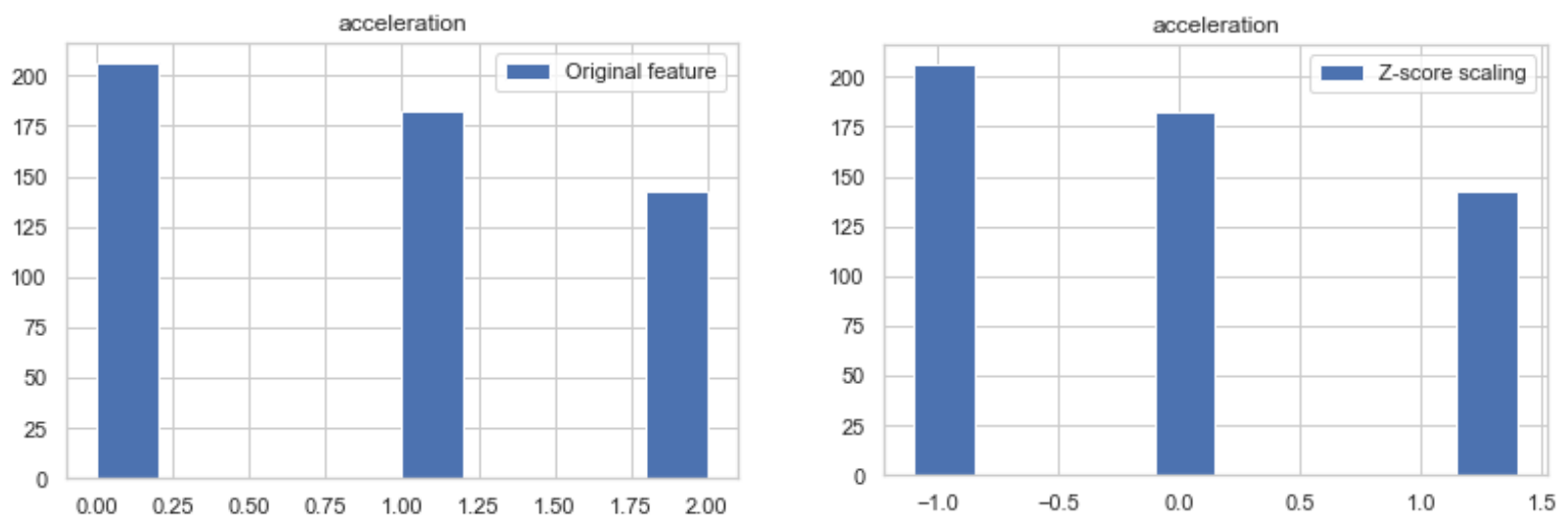

(a) Acceleration
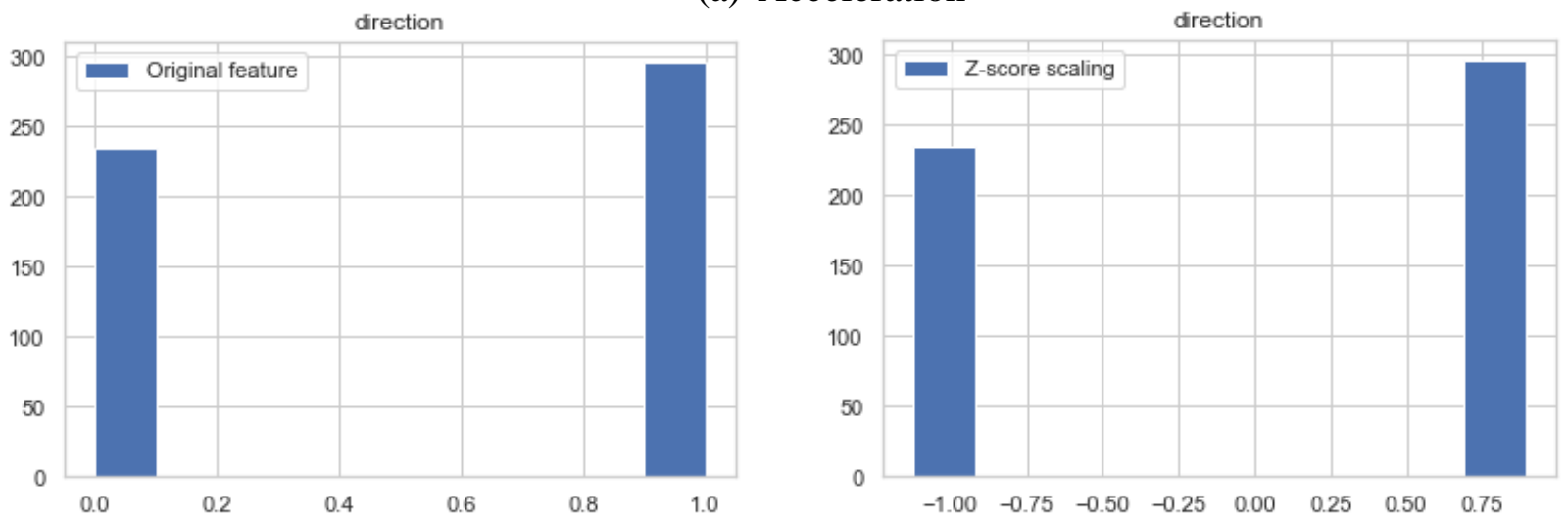

(b) Direction
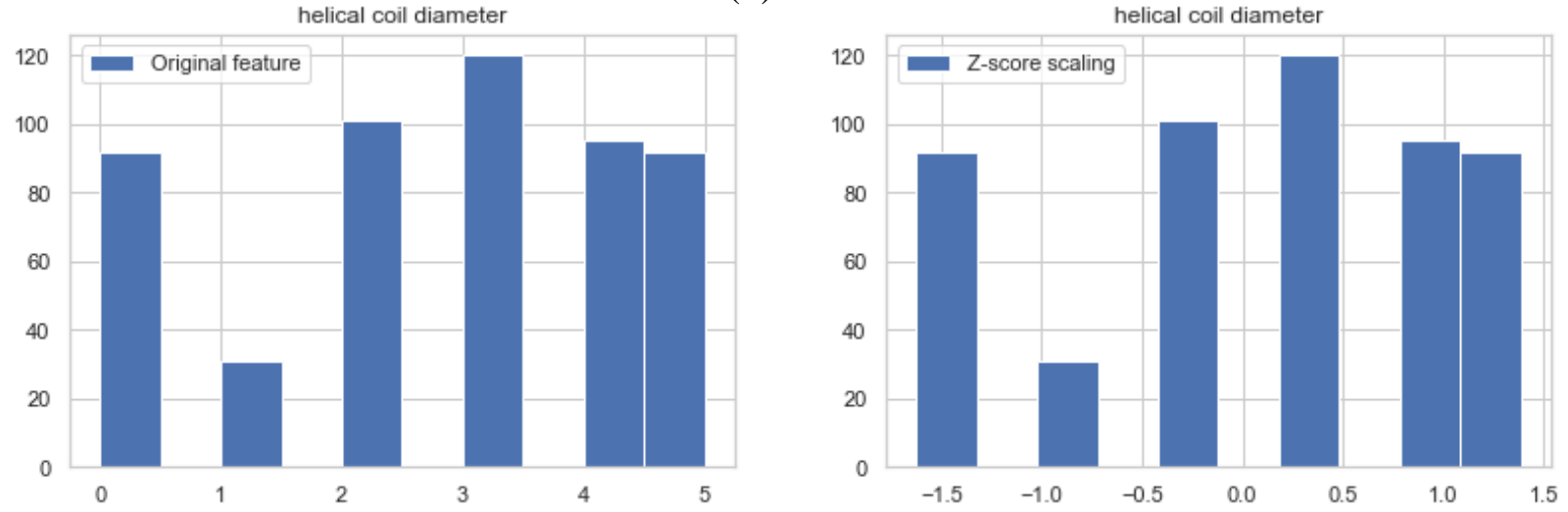

(c) Helical coil diameter
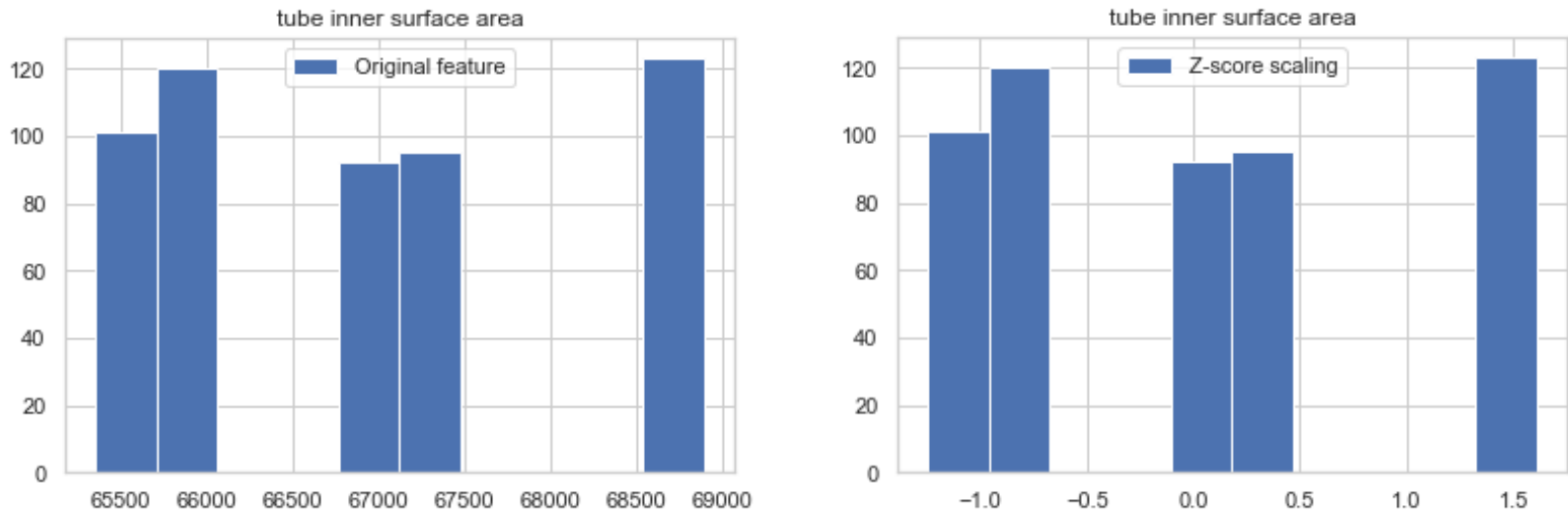

(d) Tube inner surface area

Fig. 7. Categorical input feature scaling. 

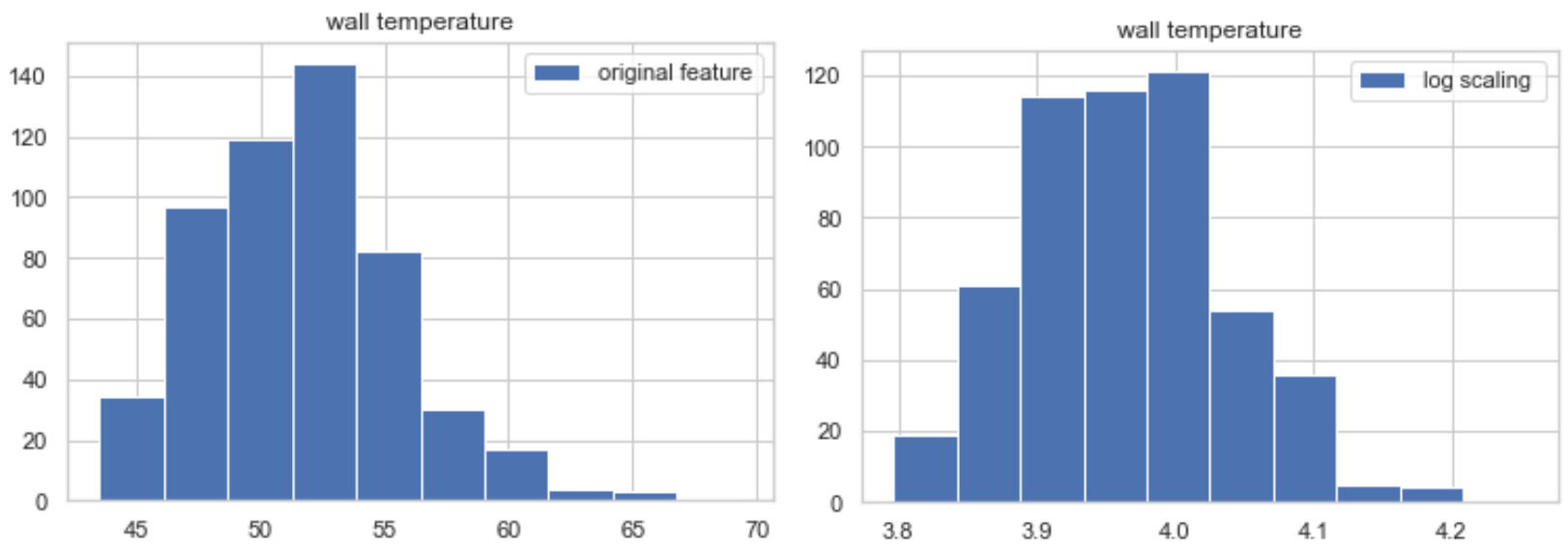

(a) Wall temperature
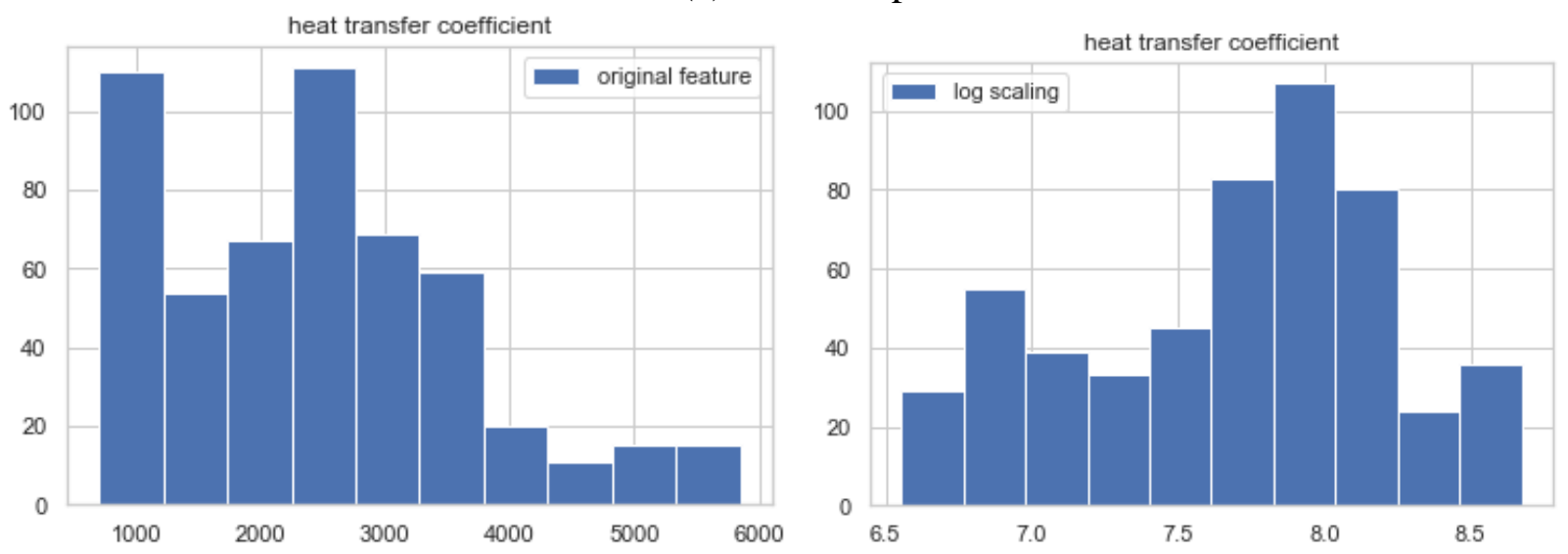

(b) Heat transfer coefficient

Fig. 8. Output feature scaling.

\subsection{Proposed model}

Among the various kinds of ANNs that exists, the standard feed-forward network has become one of the most popular solution in engineering applications. In an ANN model, the number of neurons in the input and output layers are identical to the numbers of input and output features, respectively. The number of hidden layers and neurons within each hidden layer can be varied based on the complexity of the problem and of the data set. Fig. 9 illustrates the proposed deep ANN model architecture for helical coil boiling heat transfer prediction. Similar to most existing deep learning research, the model is implemented using Python (3.7.6) Keras library (2.3.1) with Tensorflow as backend [48] on a Windows desktop with one Nvidia GeForce RTX 2080Ti adapter cards and 3.7 GHz Intel Cores i9-10900X, 10 cores, CPU with 64 GB RAM. The proposed deep ANN model comprises an input layer with eight inputs; three hidden layers with 512 nodes for the first hidden layer, 512 nodes for the second hidden layer and 64 nodes for the third hidden layer respectively; and an output layer with two outputs.

Rectified Linear Unit (ReLU) non-linear transfer function is selected for the hidden layer, and a linear transfer function is assigned to the final output layer. Model sensitivity is evaluated following the machine learning workflow illustrated in Fig. 10 for different networks with different nodes in hidden layers. During training, dropout regularisation (rate $=0.2$ ) was deployed in each hidden layer to avoid overfitting by randomly dropping $20 \%$ of the units and their connections during training. An intuitive explanation of dropout efficacy is that each unit learns to extract useful features on its own with different sets of randomly chosen inputs. As a result, each hidden unit is more robust to random fluctuations and learns a generally useful transformation. To accelerate the training process and avoid local minimums, 
we used Adam algorithm with its default parameter setting (learning rate $=0.001$, beta $1=0.9$, beta $2=0.999$ ) as the training optimizer [49].

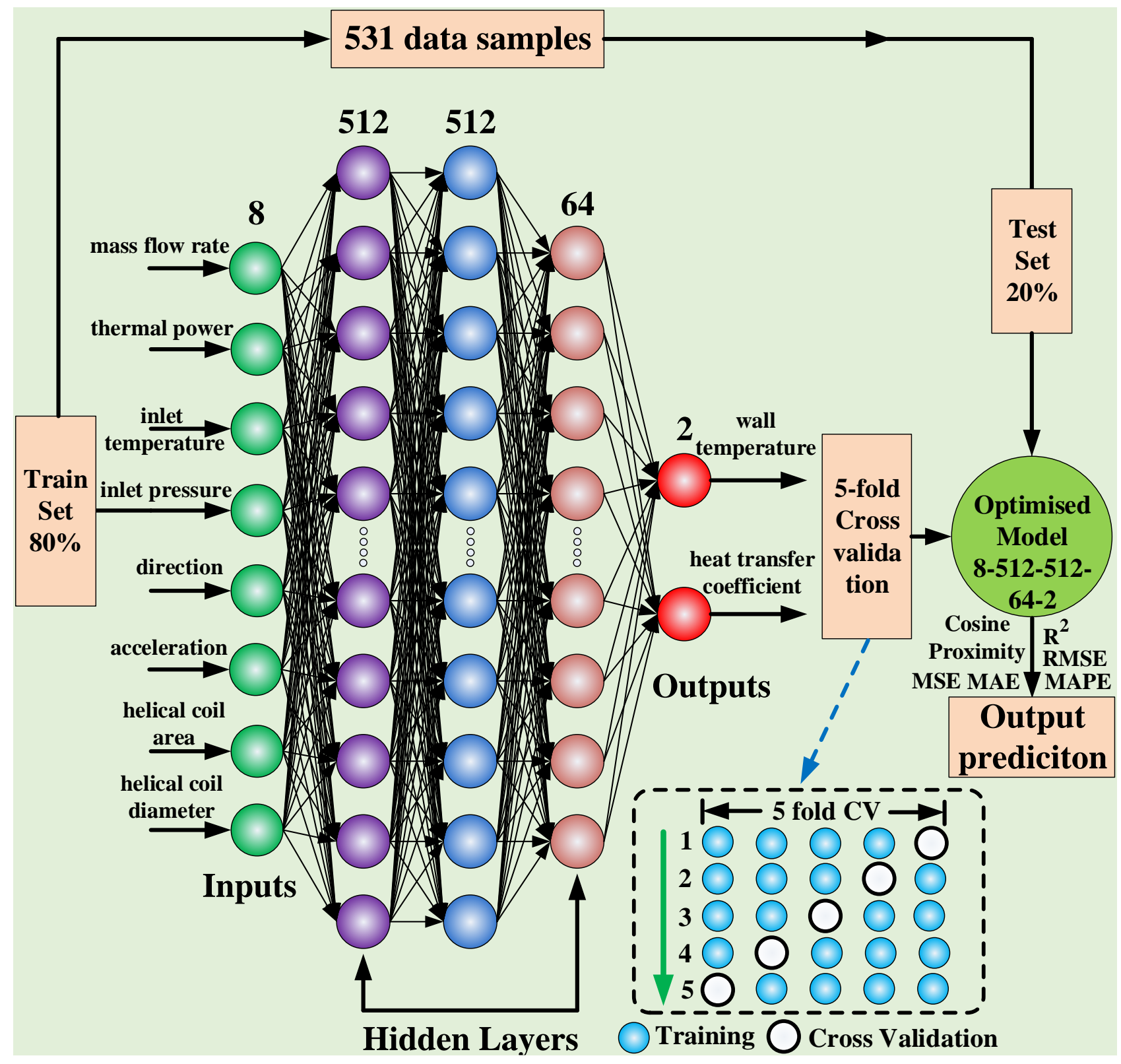

Fig. 9. Schematic diagram of the developed deep ANN model with three hidden layers.

In the present study, the model performance is obtained from each combination of the hyperparameters tuning with cross-validation (CV) methods. To avoid bias in data selection, popular k-fold CV method is employed during the process of hyperparameters tuning. As stated by [50], the bias of an accurate estimation will be smaller when the number of fold is either five or ten. In this regards, the number of folds $\mathrm{k}$ is set to five $(\mathrm{k}=5)$ as suggested by [51] associated with the trade-off between the computation time and the bias. Therefore, five rounds of train, cross-validation and test set evaluation are conducted together with random data shuffling. In summary, as shown in Fig. 9, with 5-fold CV, the entire datasets are spitted into $20 \%$ as test set, the remaining $80 \%$ for train and validation purpose (in which $80 \%$ for train set, $20 \%$ for cross-validation set). 


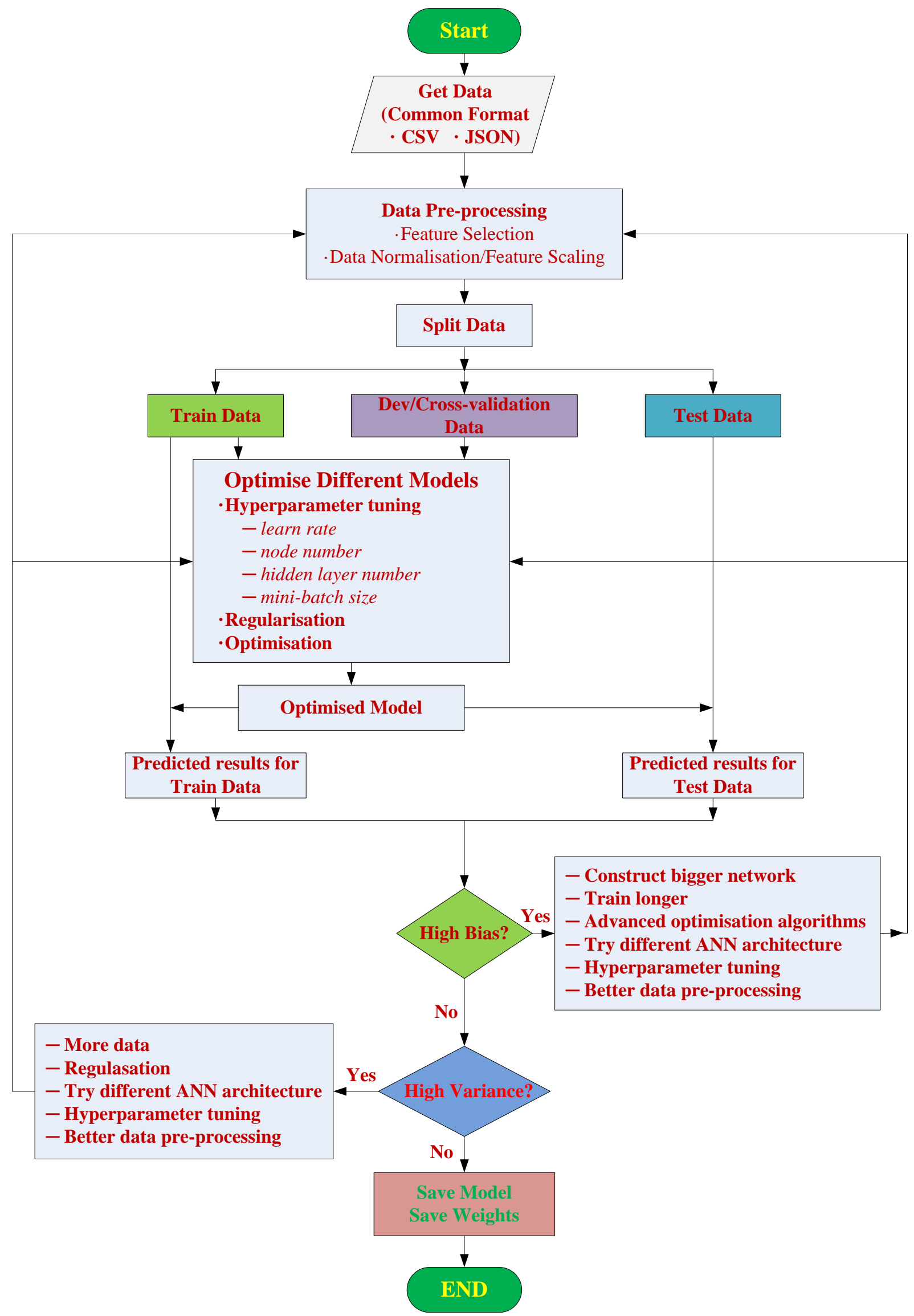

Fig. 10. Workflow for training a machine learning model. 


\section{Results and discussion}

In the present study, the 8-512-512-64-2 deep artificial neural network model with 300,266 trainable parameters in total was found to have the best performance. Table 6 summaries six verification statistics: Coefficient of Determination $\left(\mathrm{R}^{2}\right)$, Mean Squared Error (MSE), Mean Absolute Error (MAE), Mean Absolute Percentage Error (MAPE), Cosine Proximity, and Root Mean Squared Error (RMSE), which are regularly employed to analyse the predictive results on each fold and averaged results are used to present model performance.

Table 6 5-fold performance evaluation on test set.

\begin{tabular}{l|llccll}
\hline $\begin{array}{l}\mathrm{k} \text { value of } \\
\text { 5-fold }\end{array}$ & $\mathrm{R}^{2}$ & MSE & MAE & MAPE & $\begin{array}{l}\text { Cosine } \\
\text { Proximity }\end{array}$ & RMSE \\
\hline $\mathrm{k}=1$ & 0.832 & 0.018 & 0.078 & 1.174 & 1.000 & 0.142 \\
$\mathrm{k}=2$ & 0.908 & 0.012 & 0.060 & 0.919 & 1.000 & 0.117 \\
$\mathrm{k}=3$ & 0.873 & 0.013 & 0.072 & 1.075 & 1.000 & 0.116 \\
$\mathrm{k}=4$ & 0.787 & 0.030 & 0.096 & 1.386 & 1.000 & 0.178 \\
$\mathrm{k}=5$ & 0.865 & 0.016 & 0.065 & 0.996 & 1.000 & 0.126 \\
average & 0.853 & 0.018 & 0.074 & 1.110 & 1.000 & 0.136 \\
\hline
\end{tabular}

The above metrics can be defined by the following Eqs. (6) - (12). $\mathrm{R}^{2}$ metric obtained by the following Eqs. (6) and (7) indicates how well the prediction of the regression model approximate the real data points. $R^{2}$ value of 1 indicates that the regression predictions perfectly fit the data.

$$
\begin{aligned}
& y_{\text {mean }}=\frac{1}{\mathrm{n}} \sum_{k=1}^{n} y_{\text {true }} \\
& R^{2}=1-\frac{\sum_{k=1}^{n}\left(y_{\text {true }}-y_{\text {pred }}\right)^{2}}{\sum_{k=1}^{n}\left(y_{\text {true }}-y_{\text {mean }}\right)^{2}} \in[0,1] \\
& M S E=\frac{\sum_{k=1}^{n}\left(y_{\text {true }}-y_{\text {pred }}\right)^{2}}{n} \in[0,+\infty] \\
& M A E=\frac{\sum_{k=1}^{n}\left|y_{\text {true }}-y_{\text {pred }}\right|}{n} \in[0,+\infty] \\
& M A P E=\frac{1}{n} \sum_{k=1}^{n}\left|\frac{y_{\text {true }}-y_{\text {pred }}}{y_{\text {true }}}\right| \in[0,+\infty] \\
& \text { Cosine Proximity }=\frac{\sum_{k=1}^{n} y_{\text {true }_{k}} \times y_{\text {pred }_{k}}}{\sqrt{\sum_{k=1}^{n} y_{\text {true }_{k}}^{2}} \times \sqrt{\sum_{k=1}^{n} y_{\text {pred }_{k}}^{2}}} \in[-1,1] \\
& R M S E=\sqrt{\frac{\sum_{k=1}^{n}\left(y_{\text {true }}-y_{\text {pred }}\right)^{2}}{n}} \in[0,+\infty]
\end{aligned}
$$

where $y_{\text {true }}$ is the ground truth output (experimental observed output data), $y_{\text {pred }}$ is the predicted output, $y_{\text {mean }}$ is the mean of the ground truth data, $\mathrm{n}$ is the total number of data. 
MSE is the most commonly used regression loss function. It is the sum of squared distances between ground truth value and predicted value. It is always non-negative, and values closer to zero are better. MAE is the average of all absolute errors, which is another useful measure that is extensively employed in model evaluation. MAPE is the most common measure used to forecast error, and sometimes reported as a percentage, which is the above equation multiplied by 100 to make it a percentage error. Cosine proximity or cosine similarity is the measure of similarity between two vectors. A high cosine proximity indicates a higher accuracy. Perfectly opposite vectors have a cosine proximity of -1 ; perfect orthogonal vectors have a cosine proximity of 0 , and identical vectors (such as perfect fit of predicted values to the ground truth values) have a cosine proximity of 1 . RMSE is the square root of the ratio of the square of the deviation between the predicted value and ground truth value to the number of data $\mathrm{n}$. The RMSE has a value equal to or greater than 0 , where 0 depicts a statistically perfect fit to the ground truth data.

Figs. 11 and 12 plot the network training process against 1800 iterations (epochs) of the optimum network (8-512-512-64-2), in which the best result was achieved. The performance of various network configurations are compared using MSE, $\mathrm{R}^{2}$, MAE, MAPE, cosine proximity, and RMSE for regression performance evaluation.
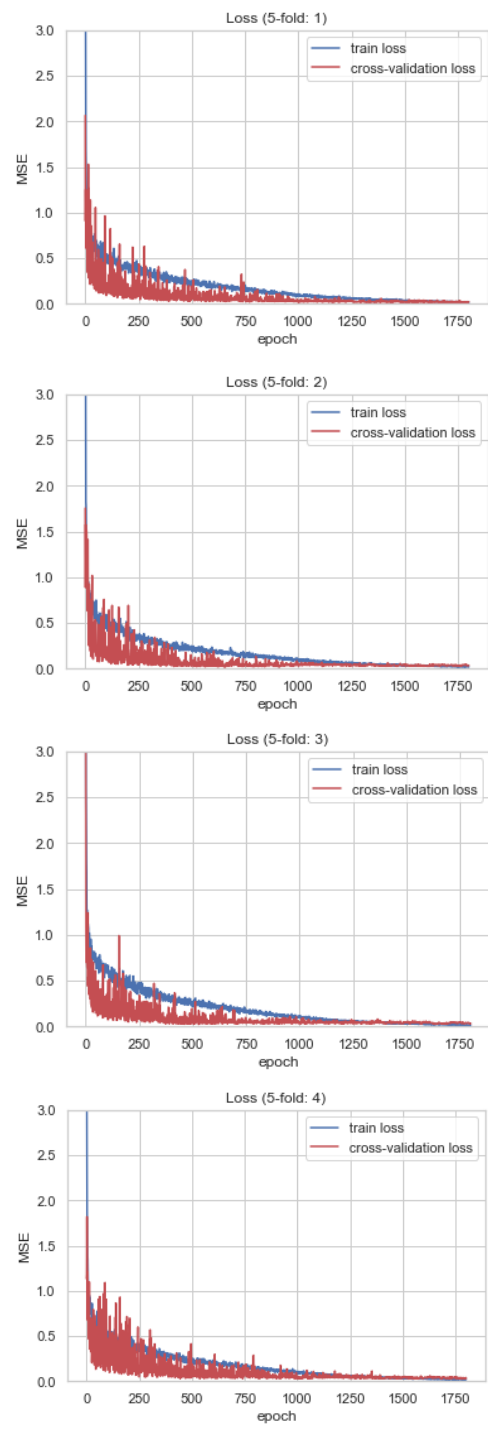
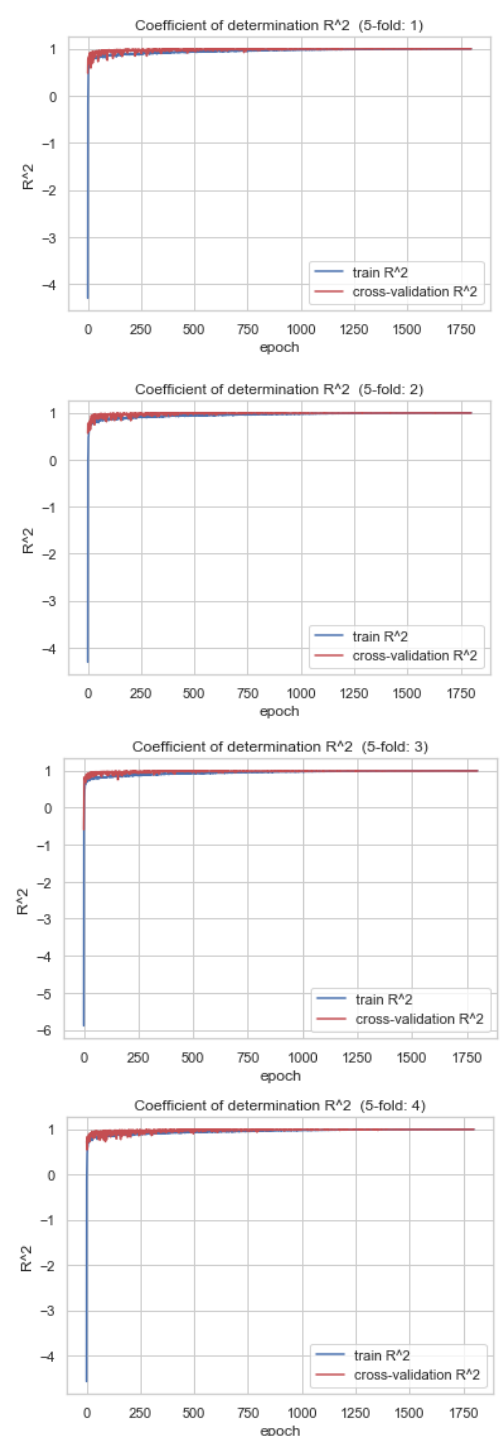
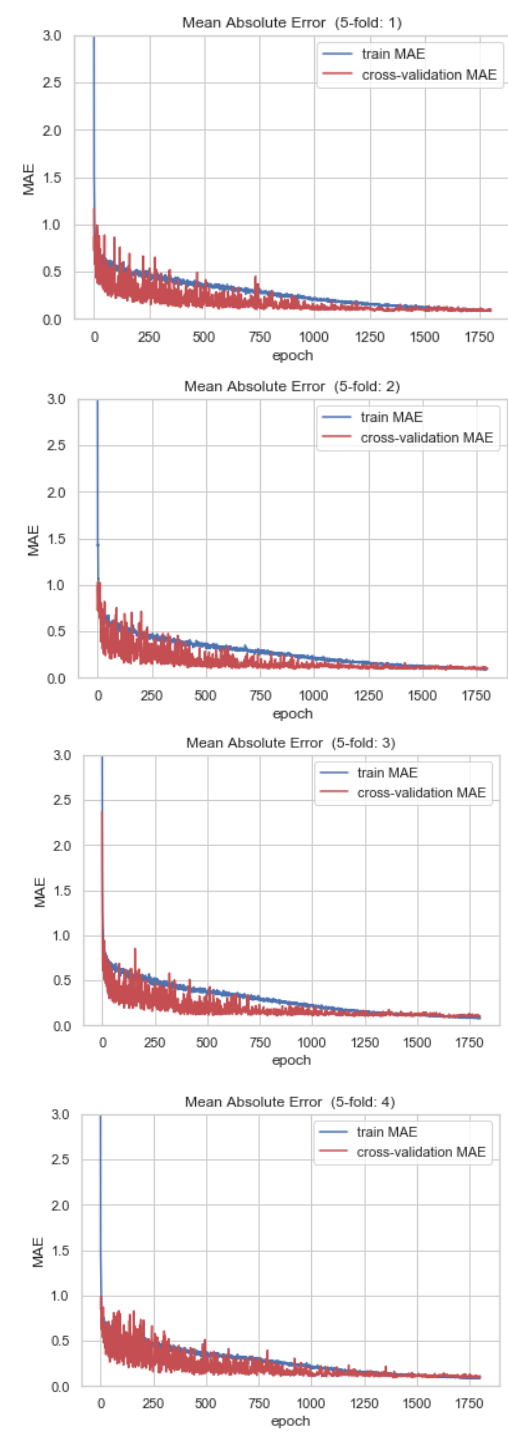

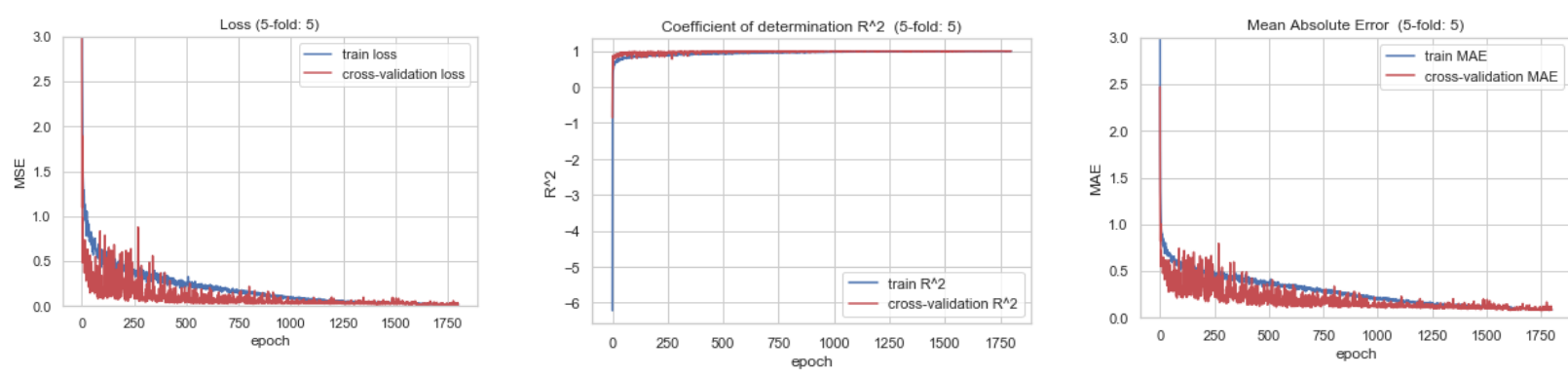

Fig. 11. Performance Evaluation on deep ANN model: MSE, ${ }^{2,}$ MAE.
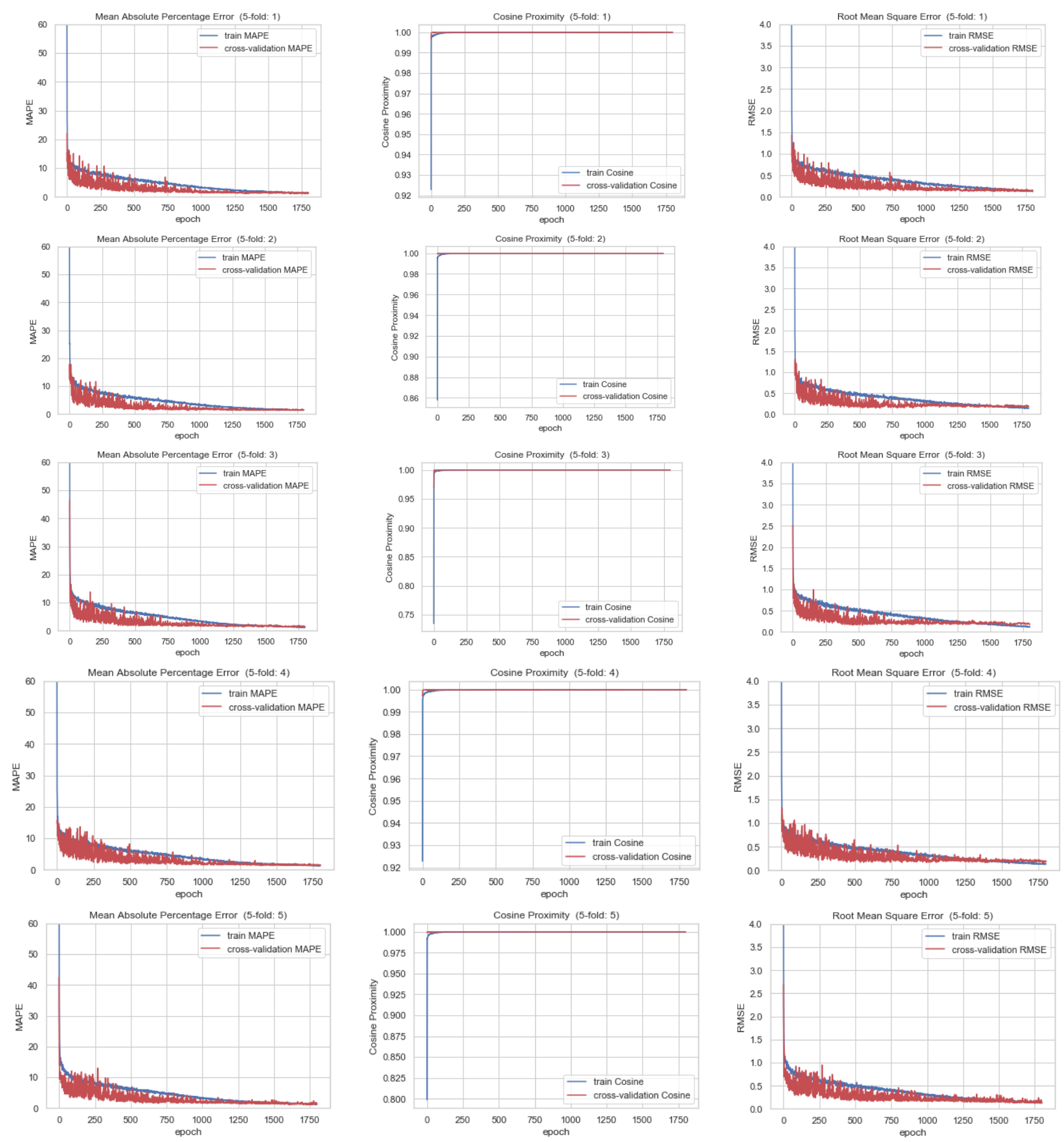

Fig. 12. Performance Evaluation on deep ANN model: MAPE, Cosine Proximity, RMSE. 
Figs. 13 and 14 show the scatter plot of the predicted values (wall temperature and heat transfer coefficient) by the developed deep ANN model against experimental values (ground truth) for the optimized model. It can be seen clearly that good accuracy between the neural network predictions and experimental data are achieved. These results can confirm the high ability of the developed deep ANN to predict the experimental data.

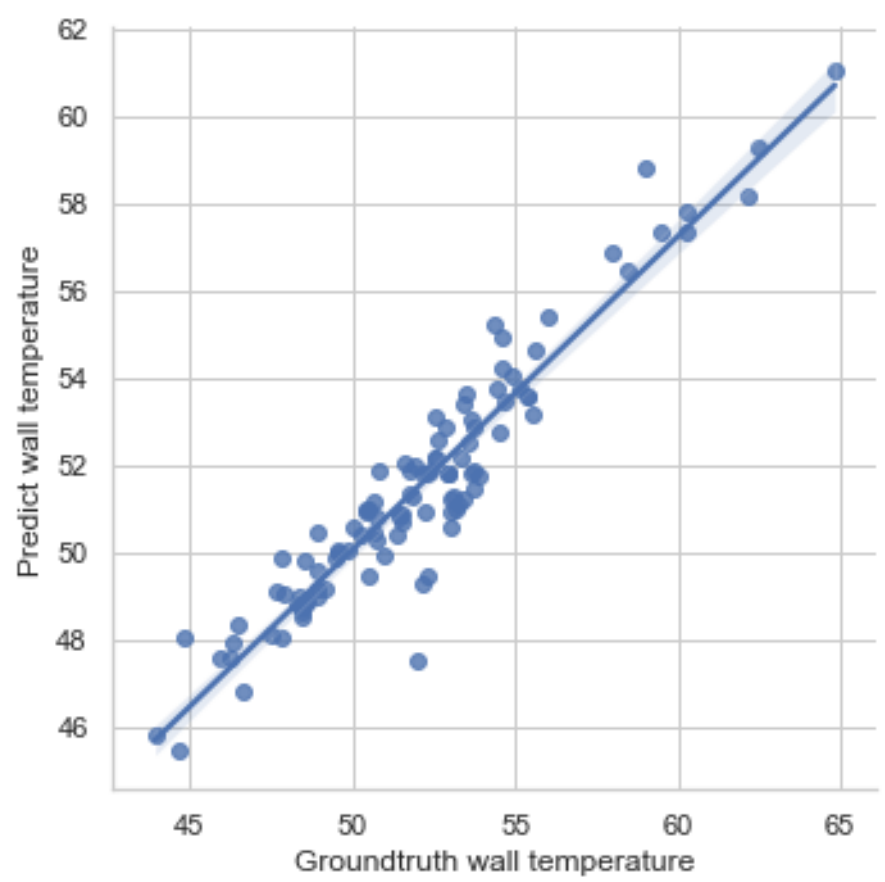

Fig. 13. Scatter plot of the predicted outlet wall temperature values by the deep ANN model versus experimental data on test set

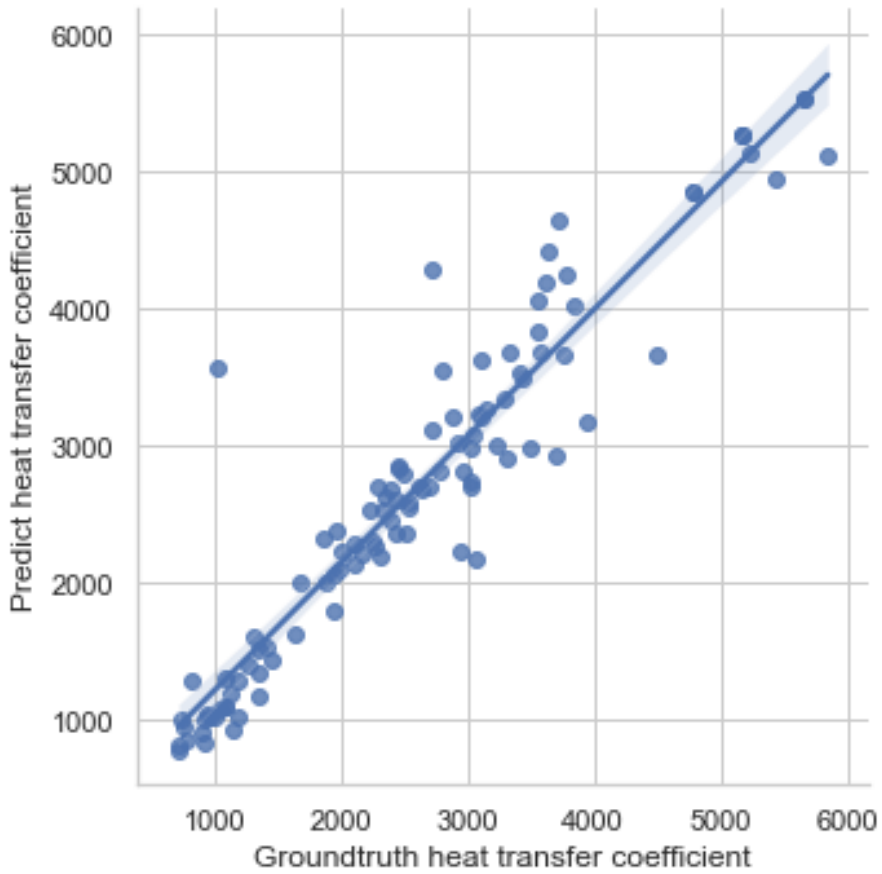

Fig. 14. Scatter plot of the predicted heat transfer coefficient values by the deep ANN model versus experimental data on test set 


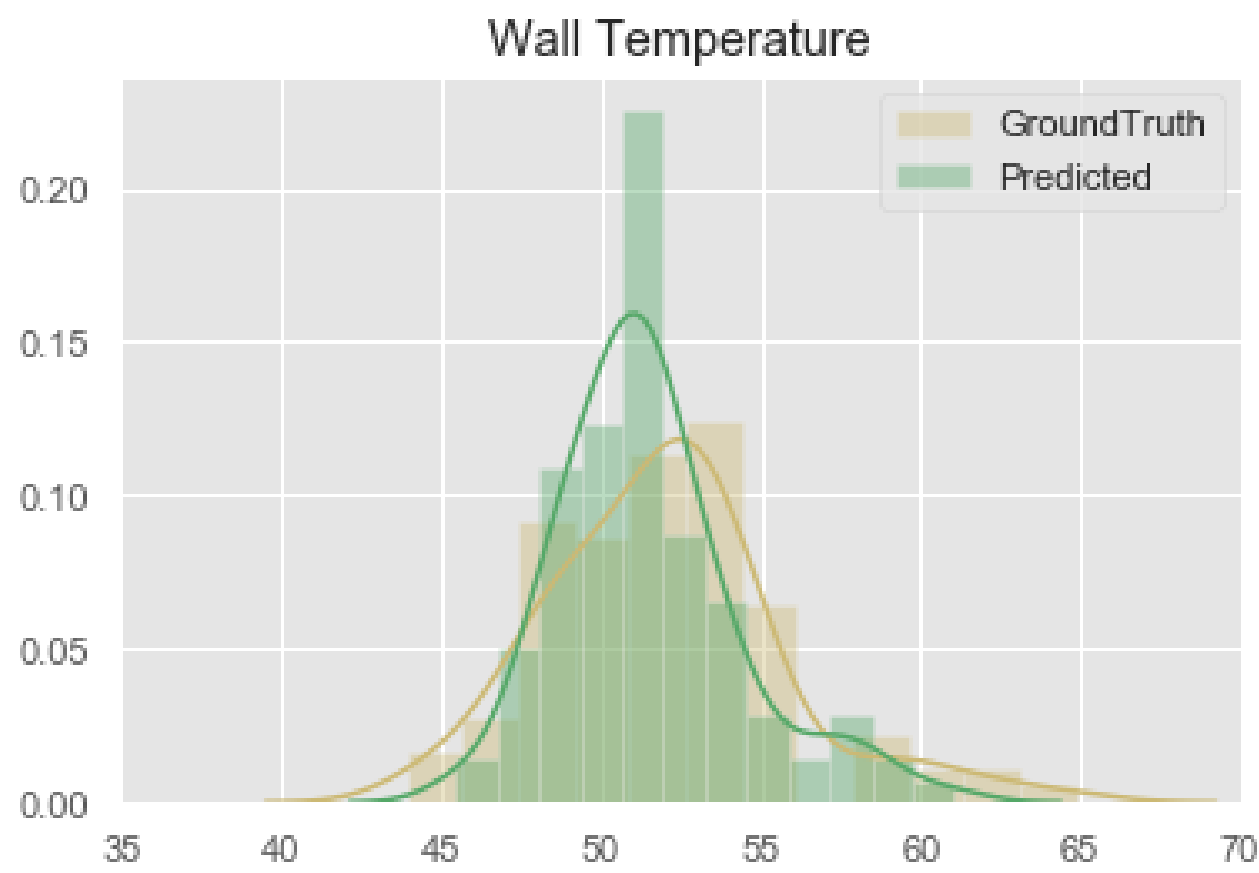

Fig. 15. KDE of wall temperature: ground truth vs. predicted.

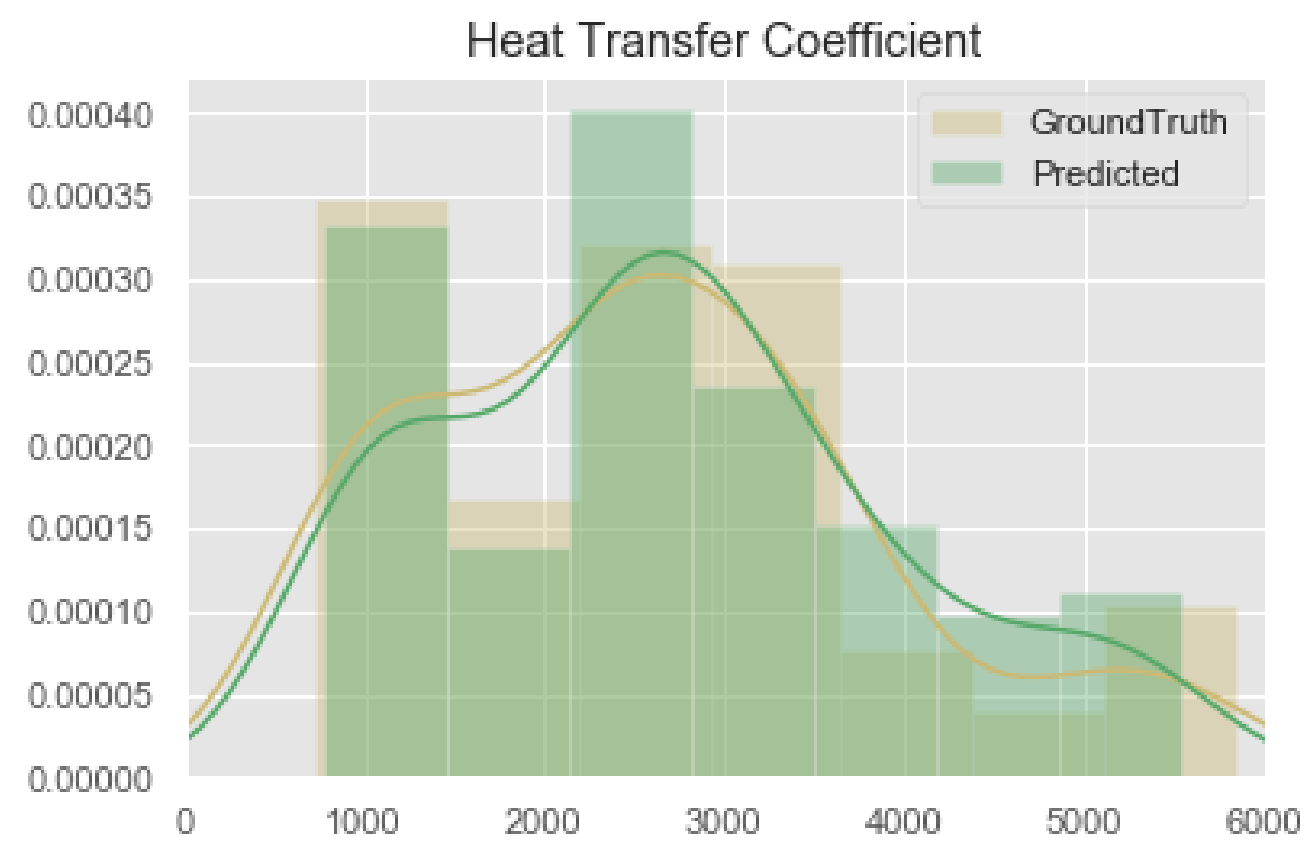

Fig. 16. KDE of heat transfer coefficient: ground truth vs. predicted.

Figs. 15 and 16 illustrate the Kerner Density Estimation (KDE) to estimate and compare the probability density distribution on test set between predicted values and ground truth values of wall temperature and heat transfer coefficient, respectively. According to performance of the network, for both wall temperature and heat transfer coefficient, the KDE probability density curve of predicted values agrees well with the one for ground truth. It is recognised that the proposed model achieves an excellent performance on test set. With the utilisation of a 4-layer deep ANN with 300,266 trainable parameters, it should be noted that the proposed model can predict the outputs in an accurate manner, more specifically, wall temperature value range: $\left(43-69^{\circ} \mathrm{C}\right)$ but heat transfer coefficient value range: $\left(701-5849 \mathrm{Wm}^{-2} \mathrm{~K}^{-1}\right)$. 


\section{CONCLUSIONS}

In this study, a deep ANN model was derived and tested for predicting the thermal performance in helical coils three different high gravity conditions. The database used for developing the deep ANN model consisting of 531 experimental datasets. An optimized deep ANN model configuration with three hidden layers and there are 512 and 512 neurons for the first two hidden layers; 64 neurons for the third hidden layer, exhibited good predictive ability with $R^{2}$ of 0.853 , MSE of 0.018 , MAE of 0.074 , MAPE of 1.110 , RMSE of 0.136 and cosine proximity of 1.000 for large range value multioutput estimation. The deep ANN model presented can easily be used without the need of assuming a mathematical model of the process. The procedure proposed here can help researchers to plan new measurements by showing where these are needed.

\section{ACKNOWLEDGMENT}

The financial supports from the Royal Academy of Engineering (DVF171818126) is gratefully acknowledged.

\section{NOMENCLATURE}

$\begin{array}{lll}A & \text { coil inner surface area } & {\left[\mathrm{mm}^{2}\right]} \\ c_{p} & \text { specific heat } & {\left[\mathrm{J} \mathrm{kg}^{-1} \mathrm{~K}^{-1}\right]} \\ h & \text { heat transfer coefficient } & {\left[\mathrm{Wm}^{-2} \mathrm{~K}^{-1}\right]} \\ i & \text { vaporisation latent heat } & {\left[\mathrm{Jkg}^{-1}\right]} \\ I & \text { DC current } & {[\mathrm{A}]} \\ \dot{m} & \text { mass flow rate } & {[\mathrm{kg} / \mathrm{s}]} \\ n & \text { number of data points } & {[-]} \\ p & \text { pressure } & {[\mathrm{kPa}]} \\ \Delta p & \text { pressure drop } & {[\mathrm{kPa}]} \\ q & \text { heat flux delivered to the fluid } & {\left[\mathrm{Wm}{ }^{-2}\right]} \\ Q_{\text {loss }} & \text { heat loss } & {[\mathrm{W}]} \\ Q_{\text {eff }} & \text { effective heating power delivered to the fluid } & {[\mathrm{W}]} \\ R^{2} & \text { coefficient of determination } & {[-]} \\ t_{c} & \text { start-up time } & {[\mathrm{min}]} \\ t_{d} & \text { acceleration duration time } & {[\mathrm{min}]} \\ T & \text { temperature } & {\left[{ }^{\circ} \mathrm{C}\right]} \\ V & \text { DC voltage } & {[\mathrm{V}]} \\ x & \text { vapour quality } & {[-]} \\ y_{\text {mean }} & \text { mean of the ground truth data } & {[-]} \\ y_{\text {pred }} & \text { predicted output } & {[-]} \\ y_{\text {true }} & \text { ground truth output } & {[-]}\end{array}$

$\begin{array}{ll}\text { Subscripts } & \\ \text { w1 } & \text { inner wall surface at outlet of helical coil } \\ \text { w2 } & \text { outer wall surface at outlet of helical coil } \\ \text { amb } & \text { ambient } \\ \text { avg } & \text { average } \\ g & \text { high acceleration } \\ \text { in } & \text { inlet } \\ \text { out } & \text { outlet } \\ \text { sat } & \text { saturated } \\ w & \text { wall }\end{array}$




\section{REFERENCES}

[1] M. Delgado, Y.A. Hassan, N.K. Anand, Experimental flow visualization study using particle image velocimetry in a helical coil steam generator with changing lateral pitch geometry, Int. J. Heat Mass Transfer 133 (2019) 756-768.

[2] H. Khosravi-Bizhaem, A. Abbassi, A.Z. Ravan, Heat transfer enhancement and pressure drop by pulsating flow through helically coiled tube: An experimental study, Appl. Therm. Eng. 160 (2019) 114012.

[3] J. Yu, J. Chen, F. Li, W. Cai, L, Lu, Y. Jiang, Experimental investigation of forced convective condensation heat transfer of hydrocarbon refrigerant in a helical tube, Appl. Therm. Eng. 129 (2018) 1634-1644.

[4] S. Zakeralhoseini, B. Sajadi, M.A.A. Behabadi, S. Azarhazin, H. Fazelnia, Experimental investigation of the heat transfer coefficient and pressure drop of R1234yf during flow condensation in helically coiled tubes, Int. J. Therm. Sci. 157 (2020) 106516.

[5] A.K. Solanki, R. Kumar, Two-phase flow condensation heat transfer characteristic of R-600a inside the horizontal smooth and dimpled helical coiled tube in shell type heat exchanger, Int. J. Refrig. 107 (2019) 155-164.

[6] A.K. Solanki, R. Kumar, Condensation frictional pressure drop characteristic of R-600a inside the horizontal smooth and dimpled helical coiled tube in shell type heat exchanger, Int. J. Therm. Sci. 154 (2020) 106406.

[7] P.C. Mukesh kumar, M. Chandrasekar, A review on helically coiled tube heat exchanger using nanofluids, Mater. Today: Proc. 21 (2020) 137-141.

[8] X. Yu, B. Sun, Y. Li, J. Shi, G. Zhang, W. Wu, Z. Zhao, Numerical investigation of thermalhydraulic parameter distribution characteristics during dryout evolution in the helically coiled oncethrough steam generator, Int. J. Heat Mass Transfer 139 (2019) 373-385.

[9] Z. Huang, Y. Zou, J. Ding, J. Lu, Experimental investigation of heat transfer in coiled tube type molten salt steam generator, Appl. Therm. Eng. 148 (2019) 1131-1138.

[10] S. Gholipour, M. Afrand, R. Kalbasi, Improving the efficiency of vacuum tube collectors using new absorbent tubes arrangement: Introducing helical coil and spiral tube adsorbent tubes, Renew. Energ. 151 (2020) 772-781.

[11] S. Akbarzaden, M.S. Valipour, Energy and exergy analysis of a parabolic trough collector using helically corrugated absorber tube, Renew. Energ. 155 (2020) 735-747.

[12] V. Mayilvelnathan, A. Valan Arasu, Experimental investigation on thermal behavior of graphene dispersed erythritol PCM in a shell and helical tube latent energy storage system, Int. J. Therm. Sci. 155 (2020) 106446.

[13] M.A. Essa, I.Y. Rofaiel, M.A. Ahmed, Experimental and theoretical analysis for the performance of evacuated tube collector integrated with helical finned heat pipes using PCM energy storage, Energy. 216 (2020) 118166.

[14] J. Gupta, M. Agarwal, A.K. Dalai, Intensified transesterification of mixture of edible and nonedible oils in reverse flow helical coil reactor for biodiesel production, Renew. Energ. 134 (2019) 509-525.

[15] S. Gul, O. Erge, E.V. Oort, Frictional pressure losses of Non-Newtonian fluids in helical pipes: Applications for automated rheology measurements, J. Nat. Gas Sci. Eng. 73 (2020) 103042.

[16] S. Chen, Z. Hu, Y. Xiao, H. Gu, Experimental investigation of subcooled flow boiling heat transfer in helical coils, Nucl. Eng. Des. 327 (2018) 187-197.

[17] M. Mansour, D. Thévenin, K. Zähringer, Numcerical study of flow mixing and heat transfer in helical pipes, coiled flow inverters and a novel coiled configuration, Chem. Eng. Sci. 221 (2020) 115690. 
[18] B.K. Hardik, S.V. Prabhu, Heat transfer distribution in helical coil flow boiling system, Int. J. Heat Mass Transfer 117 (2018) 710-728.

[19] L. Santini, A. Cioncolini, M.T. Butel, M.E. Ricotti, Flow boiling heat transfer in a helically coiled steam generator for nuclear power applications, Int. J. Heat Mass Transfer 92 (2016) 91-99.

[20] L. Tan, C. Chen, X. Dong, Z. Gong, M. Wang, Experimental study on CHF of R134a flow boiling in a horizontal helically-coiled tube near the critical pressure, Exp. Therm. Fluid Sci. 82 (2017) 472-481.

[21] L. Kong, J. Han, C. Chen, K. Xing, G. Lei, An experimental study on subcooled flow boiling heat transfer characteristics of R134a in vertical helically coiled tubes, Exp. Therm. Fluid Sci. 82 (2017) 231-239.

[22] B.K. Hardik, S.V. Prabhu, Boiling pressure drop and local heat transfer distribution of helical coils with water at low pressure, Int. J. Therm. Sci. 114 (2017) 44-63.

[23] B.K. Hardik, S.V. Prabhu, Boiling pressure drop, local heat transfer distribution and critical heat flux in helical coils with R123, Int. J. Therm. Sci. 125 (2018) 149-165.

[24] Y. Xiao, Z. Hu, S. Chen, H. Gu, Experimental investigation of boiling heat transfer in helically coiled tubes at high pressure, Ann. Nucl. Energy 113 (2018) 409-419.

[25] X. Niu, H. Yuan, C. Quan, L. Zhao, Dryout quality prediction for boiling two-phase flow in vertically helically coiled tubes, Appl. Therm. Eng. 128 (2018) 982-992.

[26] B. Sun, X., Yu, S. Liu, J. Shi, L. Yang, G. Zhang, P. Zhang, Non-uniform wall temperature distribution of nucleate boiling heat transfer in helically coiled tubes, Nucl. Eng. Des. 330 (2018) 356-367.

[27] J.A. Hernández-Magallanes, W. Rivera, Boiling heat transfer coefficients in a falling film helical coil heat exchanger for the NH3-LiNo3 mixture, J. Heat Transfer 141 (2019) 071502.

[28] D.R. Shaver, N. Salpeter, A. Tomboulides, P. Vegendla, A. Tentner, W. David Pointer, E. Merzari, Simulation of boiling two-phase flow in a helical coil steam generator using the spectral element code Nek-2P, Nucl. Technol. 206 (2020) 375-387.

[29] L. Xie, Y. Xie, H. Wu, J. Yu, High gravity influence on boiling heat transfer in helical coils, Int. J. Heat Mass Transfer 73 (2014) 706-715.

[30] L. Xie, Y. Xie, J. Yu, Phase distributions of boiling flow in helical coils in high gravity, Int. J. Heat Mass Transfer 80 (2015) 7-15.

[31] W. Bai, S. Zhang, H. Li, X. Xu, Effects of abnormal gravity on heat transfer of supercritical CO2 in heated helically coiled tube, Appl. Therm. Eng. 159 (2019) 113833.

[32] Y. Yang, P., Perdikaris, Adversarial uncertainty quantification in physics-informed neural networks. J. Comput. Phys., 394 (2019) 136-152.

[33] M. Raissi, P. Perdikaris, G.E. Karniadakis, Physics-informed neural networks: A deep learning framework for solving forward and inverse problems involving nonlinear partial differential equations. J. Comput. Phys., 378 (2019) 686-707.

[34] A.D. Jagtap, K. Kawaguchi, G.E. Karniadakis. Adaptive Activation Functions Accelerate Convergence in Deep and Physics-informed Neural Networks. J. Comput. Phys., 404 (2020) 109136.

[35] M. Ravichandran, M. Bucci, Online, quasi-real-time analysis of high-resolution, infrared, boiling heat transfer investigations using artificial neural networks, Appl. Therm. Eng. 163 (2019) 114357.

[36] G.M. Hobold, A.K. da Silva, Automatic detection of the onset of film boiling using convolutional neural networks and Bayesian statistics, Int. J. Heat Mass Transfer 134 (2019) 262-270.

[37] G.M. Hobold, A.K. da Silva, Visualization-based nucleate boiling heat flux quantification using maching learning, Int. J. Heat Mass Transfer 134 (2019) 511-520.

[38] Y. Qiu, D. Garg, L. Zhou, C.R. Kharangate, S-M., Kim, I. Mudawar, An artificial neural network model to predict mini/micro-channels saturated flow boiling heat transfer coefficient based on universal consolidated data, Int. J. Heat Mass Transfer 149 (2020) 119211.

[39] A.M. Bahman, S.A. Ebrahim, Prediction of the minimum film boiling temperature using artificial neural network, Int. J. Heat Mass Transfer 155 (2020) 119834. 
[40] F. Liang, J. Gao, L. Xu, Thermal performance investigation of the miniature revolving heat pipes using artificial neural networks and genetic algorithms, Int. J. Heat Mass Transfer 151 (2020) 119394. [41] J. Gill, J. Singh, Use of artificial neural network approach for depicting mass flow rate of R134a/LPG refrigerant through straight and helical coiled adiabatic capillary tubes of vapor compression refrigeration system, Int. J. Refrig. 86 (2018) 223-238.

[42] A. Parrales, D. Colorado, J.A. Díaz-Gómez, A. Huicochea, A. Álvarez, J.A. Hernández, New void fraction equations for two-phase flow in helical heat exchangers using artificial neural networks, Appl. Therm. Eng. 130 (2018) 149-160.

[43] M. Izadi, M. Rahimi, R. Beigzadeh, Evaluation of micromixing in helically coiled microreactors using artificial intelligence approaches, Chem. Eng. J. 356 (2019) 570-579.

[44] A. Baghban, M., Kahani, M.A. Nazari, M.H. Ahmadi, W-M, Yan, Sensitivity analysis and application of machine learning methods to predict the heat transfer performance of CNT/water nanofluid flows through coils, Int. J. Heat Mass Transfer 128 (2019) 825-835.

[45] K. Sharifi, M. Sabeti, M. Rafiei, A.H. Mohammadi, A. Ghaffari, M.H. Asl, H. Yousefi, A good contribution of computational fluid dynamics (CFD) and GA-ANN methods to find the best type of helical wire inserted tube in heat exchangers, Int. J. Therm. Sci. 154 (2020) 106398.

[46] E.W. Lemmon, M.L. Hube, M.O. McLinden, Physical and chemical properties division, REFPROP 8.0, NIST Standard Reference Database 23, Version 8.0.

[47] R.J. Moffat, Describing the uncertainties in experimental results, Exp. Therm. Fluid Sci. 1 (1988) 3-17.

[48] F. Chollet, Keras: https://keras.io (2015).

[49] D. Kingma, J. Ba, Adam: A method for stochastic optimization. In: Proceedings of International Conference on Learning Representations (2015).

[50] J.D. Rodriguez, A. Perez, J.A. Lozano, Sensitivity analysis of k-fold cross validation in prediction error estimation. IEEE Trans. Pattern Anal. Mach. Intell. 32 (2010) 569-575.

[51] T.T. Wong, Performance evaluation of classification algorithms by k-fold and leave-one-out cross validation. Pattern Recognit. 48 (2015) 2839-2846. 